\title{
Mimics in chest disease: interstitial opacities
}

\author{
Anastasia Oikonomou • Panos Prassopoulos
}

Received: 19 June 2012 / Revised: 1 October 2012 / Accepted: 16 November 2012 /Published online: 18 December 2012

(C) The Author(s) 2012. This article is published with open access at Springerlink.com

\begin{abstract}
Septal, reticular, nodular, reticulonodular, groundglass, crazy paving, cystic, ground-glass with reticular, cystic with ground-glass, decreased and mosaic attenuation pattern characterise interstitial lung diseases on high-resolution computed tomography (HRCT). Occasionally different entities mimic each other, either because they share identical HRCT findings or because of superimposition of patterns. Idiopathic pulmonary fibrosis (IPF), fibrosis associated with connective tissue disease, asbestosis, end-stage sarcoidosis or chronic hypersensitivity pneumonitis (HP) may present with lower zone, subpleural reticular pattern associated with honeycombing. Lymphangiomyomatosis may be indistinguishable from histiocytosis or extensive emphysema. Both pulmonary oedema and lymphangitic carcinomatosis may be characterised by septal pattern resulting from thickened interlobular septa. Illdefined centrilobular nodular pattern may be identically present in HP and respiratory bronchiolitis-associated with interstitial lung disease (RBILD). Sarcoidosis may mimic miliary tuberculosis or haematogenous metastases presenting with miliary pattern, while endobronchial spread of tuberculosis may be indistinguishable from panbronchiolitis, both presenting with tree-in-bud pattern. Atypical infection presenting with ground-glass mimics haemorrhage. Ground-glass pattern with minimal reticulation is seen in desquamative interstitial pneumonia (DIP), RBILD and non-specific interstitial pneumonia (NSIP). Obliterative bronchiolitis and panlobular emphysema may present with decreased attenuation pattern, while obliterative bronchiolitis, chronic pulmonary embolism and HP may manifest with mosaic attenuation pattern. Various
\end{abstract}

\footnotetext{
A. Oikonomou $(\bowtie) \cdot$ P. Prassopoulos

Department of Radiology, University Hospital of Alexandroupolis, Democritus University of Thrace, Dragana, 68100 Alexandroupolis, Greece

e-mail: aoikonom@med.duth.gr

P. Prassopoulos

e-mail: pprasopo@med.duth.gr
}

mimics in interstitial lung diseases exist. Differential diagnosis is narrowed based on integration of predominant HRCT pattern and clinical history.

Teaching Points

- To learn about the different HRCT patterns, which are related to interstitial lung diseases.

- To be familiar with the more "classical" entities presenting with each HRCT pattern.

- To discuss possible overlap of different HRCT patterns and the more common mimics in each case.

- To learn about some clues that help differentiate the various diagnostic mimics on HRCT.

Keywords Interstitial lung diseases $\cdot$ HRCT patterns · Mimics $\cdot$ Reticular $\cdot$ Nodular $\cdot$ Ground-glass opacity $\cdot$ Cystic

\section{Introduction}

Since the advent of high-resolution computed tomography (HRCT) in the 1990s the understanding of interstitial lung disease has dramatically changed. As CT descriptions of the various diffuse lung diseases are being continuously refined and certain CT characteristics are considered pathognomonic while others become less specific, HRCT finds its role in an integrated approach of diagnosis where it provides sufficient information to produce a differential diagnosis and in many cases allows a non-invasive definitive diagnosis [1].

There are various patterns of interstitial lung disease on HRCT, each one representing different interstitial lung diseases with completely different histological appearances and clinical manifestations. The main distinct interstitial HRCT patterns are five: septal, reticular, nodular, cystic, groundglass and decreased lung attenuation pattern. Although each HRCT pattern encompasses many different interstitial lung entities, the contrary may also occur, namely one entity may 
present with many different patterns and have many different "faces". To make things even more complicated, there may be overlap of HRCT patterns in one entity. In everyday clinical practice one should try to combine the different HRCT patterns or identify the predominant pattern in order to make the correct diagnosis $[2,3]$. Integration of HRCT findings with clinical findings and the knowledge of acute or chronic symptoms is crucial to reach the correct diagnosis.

\section{HRCT patterns}

The most common HRCT patterns include the septal, reticular, ground-glass opacity, crazy paving pattern, mixed ground-glass opacity and reticular, nodular tree-in-bud pattern, nodular without tree-in-bud pattern, nodular with illdefined centrilobular, reticulonodular, cystic, mixed cystic with ground-glass opacity, decreased attenuation and mosaic attenuation pattern (Table 1).

For the easier approach to this review by the reader, we describe a "classic" entity and the various "mimics" for each HRCT pattern. The "classic" entity is selected in an arbitrary way either because it may be the most representative and commonly one reported for the specific HRCT pattern or because it may be the entity for which, the specific HRCT pattern was initially described.

Septal pattern

Septal pattern is defined as thickening of the interlobular septae (i.e. the borders of the secondary pulmonary lobules). Normally very few interlobular septae are seen in the anterior and lower aspects of the lower lobes on HRCT. Thickened interlobular septae are demonstrated as short lines extending perpendicularly to the peripheral pleura or the fissures, or as polygonal arcades surrounding secondary pulmonary lobules more centrally. Septal thickening can be smooth, nodular or irregular [4].

Classic entity The prototype disease entity with smooth septal thickening is hydrostatic pulmonary oedema (Fig. 1). HRCT is usually not performed to diagnose pulmonary oedema, since the diagnosis is based on clinical and radiographic findings, but it may rarely be performed upon discrepancy between the clinical history and the chest radiograph [5]. It has a perihilar and lower lobe predominance and it is usually associated with areas of ground-glass opacity and consolidation. Pleural effusion —usually bilateral, symmetric

Table 1 HRCT patterns and differential diagnosis of interstitial lung diseases

\begin{tabular}{|c|c|c|c|}
\hline \multicolumn{3}{|c|}{ HRCT PATTERN } & DIFFERENTIAL DIAGNOSIS OF DISEASES \\
\hline \multicolumn{3}{|l|}{ Septal } & $\begin{array}{l}\text { Hydrostatic pulmonary oedema, lymphangitic carcinomatosis, sarcoidosis, } \\
\text { Niemann-Pick disease, Erdheim-Chester disease, cystic lymphangiectasia, } \\
\text { pulmonary lymphangiomatosis }\end{array}$ \\
\hline \multicolumn{3}{|c|}{ Reticular } & IPF, NSIP, asbestosis, chronic HP, rheumatoid arthritis, DIP, end-stage sarcoidosis \\
\hline \multicolumn{3}{|l|}{ GGO } & Subacute HP, RBILD, DIP, Pneumocystis pneumonia \\
\hline \multicolumn{3}{|c|}{ Crazy paving pattern } & $\begin{array}{l}\text { Alveolar proteinosis, Pneumocystis pneumonia, exogenous lipoid pneumonia, } \\
\text { sarcoidosis, diffuse alveolar haemorrhage, viral/opportunistic infection, } \\
\text { invasive mucinous adenocarcinoma }\end{array}$ \\
\hline \multicolumn{3}{|c|}{ Mixed GGO and reticular } & NSIP, scleroderma, IPF, DIP \\
\hline \multirow[t]{5}{*}{ Nodular } & Random & & Haematogenous metastases, miliary infection \\
\hline & Perilymphatic & & Sarcoidosis, silicosis, coal workers pneumoconiosis \\
\hline & Centrilobular & without tree-in-bud & silicosis, coal workers pneumoconiosis \\
\hline & & with tree-in-bud & $\begin{array}{l}\text { Panbronchiolitis, tuberculosis, atypical mycobecteria infection, metastatic } \\
\text { tumour emboli }\end{array}$ \\
\hline & & ill-defined & $\begin{array}{l}\text { Subacute HP, RBILD, cryptogenic organising pneumonia, lymphocytic interstitial } \\
\text { pneumonia, follicular bronchiolitis }\end{array}$ \\
\hline \multicolumn{3}{|c|}{ Reticulonodular } & Sarcoidosis, berylliosis, lymphangitic carcinomatosis \\
\hline \multicolumn{3}{|l|}{ Cystic } & $\begin{array}{l}\text { Lymphangioleiomyomatosis, pulmonary histiocytosis, lymphocytic interstitial } \\
\text { pneumonia, centrilobular emphysema }\end{array}$ \\
\hline \multicolumn{3}{|c|}{ Mixed Cystic and GGO } & Pneumocystis pneumonia, lymphocytic interstitial pneumonia, subacute HP, DIP \\
\hline \multicolumn{3}{|c|}{ Decreased attenuation } & Obliterative bronchiolitis, panlobular emphysema \\
\hline \multicolumn{3}{|c|}{ Mosaic attenuation } & $\begin{array}{l}\text { Obliterative bronchiolitis, chronic thromboembolic pulmonary hypertension, } \\
\text { subacute HP, RBILD }\end{array}$ \\
\hline
\end{tabular}

$D I P$ desquamative interstitial pneumonia, $G G O$ ground-glass opacity, $H P$ hypersensitivity pneumonitis, IPF idiopathic pulmonary fibrosis, NSIP non-specific interstitial pneumonia, RBILD respiratory bronchiolitis-associated with interstitial lung disease 


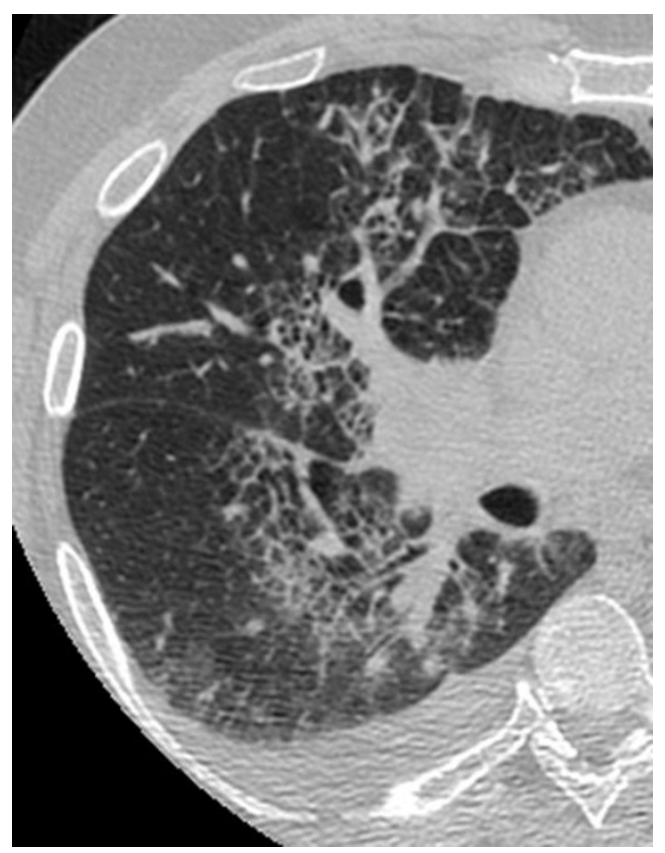

Fig. 1 Hydrostatic pulmonary oedema secondary to endocarditis and rupture of a leaflet of the mitral valve. HRCT scan of the right lung shows a "septal pattern" characterised by thickened smoothly interlobular septae in the right parahilar area. Right pleural effusion is also seen

and extensive- is a common finding. Key findings in hydrostatic pulmonary oedema are cardiomegaly, dilatation of pulmonary veins, peribronchovascular thickening and lower lobe predominance. However, pulmonary oedema may occur in the absence of an enlarged heart silhouette in cases of mitral reflux, left atrial enlargement and elevated pulmonary artery pressure [2].

Mimics There are many "mimics" of the septal pattern: lymphangitic carcinomatosis, sarcoidosis and Niemann-Pick disease, Erdheim-Chester disease, cystic lymphangiectasia and occasionally pulmonary fibrosis.

Lymphangitic carcinomatosis is characterised by nodular - and less commonly by smooth - thickening of any part of the peribronchovascular interstitium, apart from the interlobular septal thickening (i.e. intralobular interstitium, centrilobular interstitium, fissures). Lymphangitic carcinomatosis may be caused by pulmonary or extrapulmonary neoplasms (breast, pancreas, gastrointestinal, prostate cancer). When it results from lung cancer it may be focal and unilateral, whereas when it results from extrapulmonary neoplasms it is usually bilateral and symmetric. Lymphangitic carcinomatosis may be central or peripheral. Pleural effusion and lymphadenopathy is seen in 30 and $40 \%$ of cases, respectively [6] (Fig. 2).

Mimic of nodular or smooth thickening of the interlobular septae is sarcoidosis. Actually sarcoidosis is a great mimic of any pattern since it may present with many different forms. When it is manifested with septal pattern — as the predominant pattern-it may also exhibit intralobular thickening, linear opacities and perilymphatic distribution of micronodules that may add to the nodular thickening of the interlobular septae $[7,8]$. There is usually an upper/middle and central/perihilar predominance in sarcoidosis (Fig. 3). The presence of beading of the fissures (due to the perilymphatic distribution of the micronodules) and the presence of mediastinal lymphadenopathy that may occasionally have the characteristic "icing sugar" calcification are the key findings in the diagnosis of sarcoidosis [9].

Niemmann-Pick disease is an uncommon mimic of the smooth septal pattern which results in extensive, bilateral and symmetric thickening of the interlobular septae [10] (Fig. 4). Key findings may be the extensive distribution of abnormalities and the absence of cardiomegaly, lymphadenopathy or beading of the fissures. Splenomegaly is often observed in the upper abdomen (Fig. 4). Erdheim-Chester disease [11], cystic lymphangiectasia [12] and pulmonary lymphangiomatosis [13] are three other uncommon mimics of smooth septal pattern. Irregular thickening of the interlobular septae may be seen in pulmonary fibrosis but this rather constitutes an ancillary finding instead of the predominant pattern.

\section{Reticular pattern}

On HRCT, reticular pattern is characterised by innumerable interlacing shadows suggesting a mesh. The constituents of

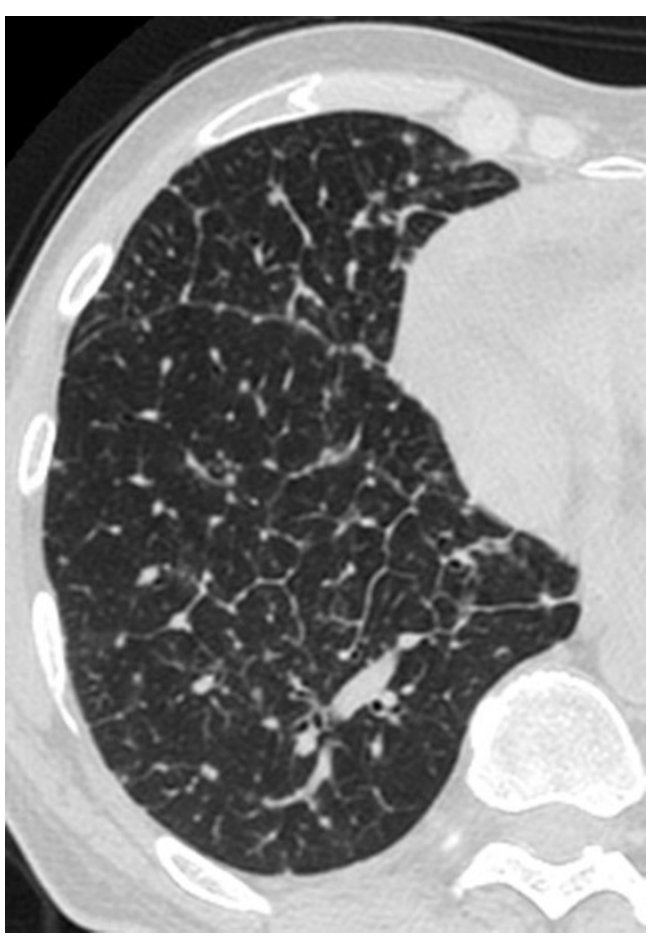

Fig. 2 Lymphangitic carcinomatosis. HRCT of the right lung shows a "septal pattern" characterised by diffuse nodular thickening of the interlobular septae and the right major fissure 


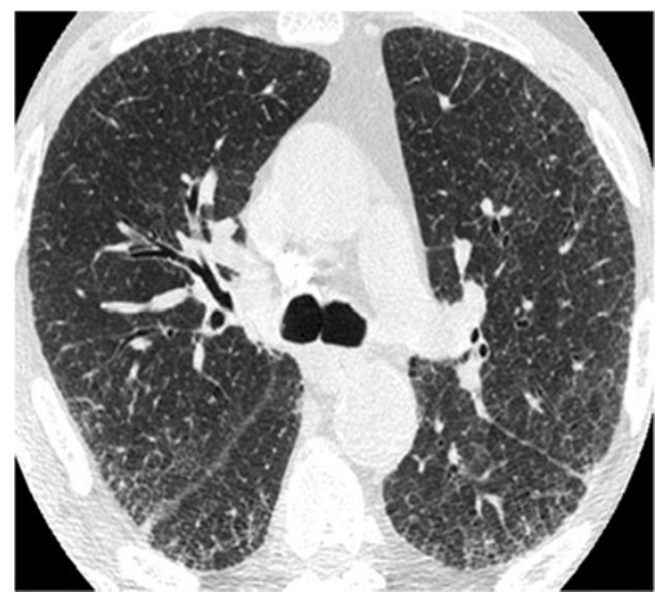

Fig. 3 Sarcoidosis. HRCT at the level of the upper lobes shows "septal pattern" characterised by mild thickening of the interlobular septae predominantly in the peripheral and subpleural posterior areas of the lungs. A calcified right paratracheal lymphnode is also noted

the reticular pattern may be all or some of the following: interlobular septal thickening, intralobular interstitial thickening, wall cysts of honeycombing, peribronchovascular interstitial thickening and traction bronchiectasis/ bronchiolectasis [4].

Classic entity The prototype entity for reticular pattern is idiopathic pulmonary fibrosis, which is characterised by subpleural and posterior predominance of intralobular interstitial thickening, traction bronchiectasis/bronchiolectasis, irregular interlobular septal thickening and honeycombing. Areas of ground-glass opacity associated with bronchiectasis and bronchiolectasis may also coexist, but areas of pure ground-glass opacity are extremely rare.

Key findings for idiopathic pulmonary findings are the lower, posterior and subpleural predominance and the presence of honeycombing [14] (Fig. 5).

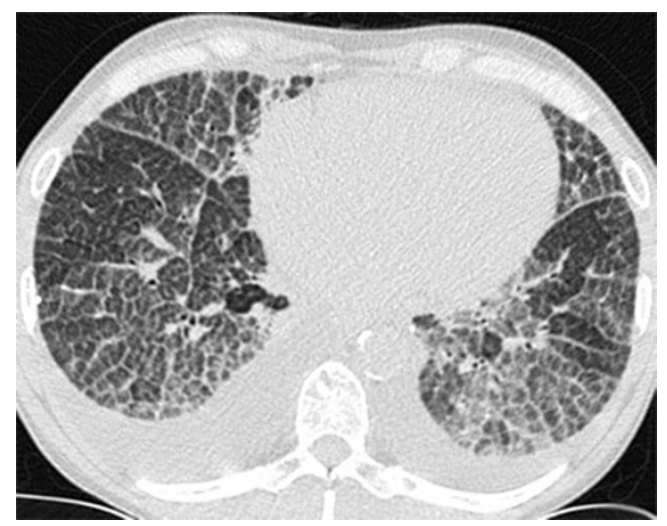

Fig. 4 Niemann-Pick. HRCT at the level of the lower lobes shows intense and homogenous thickening of the interlobular septae diffusely in the lungs. Superimposed areas of ground-glass opacity are also noted. Note also bilateral pleural effusions

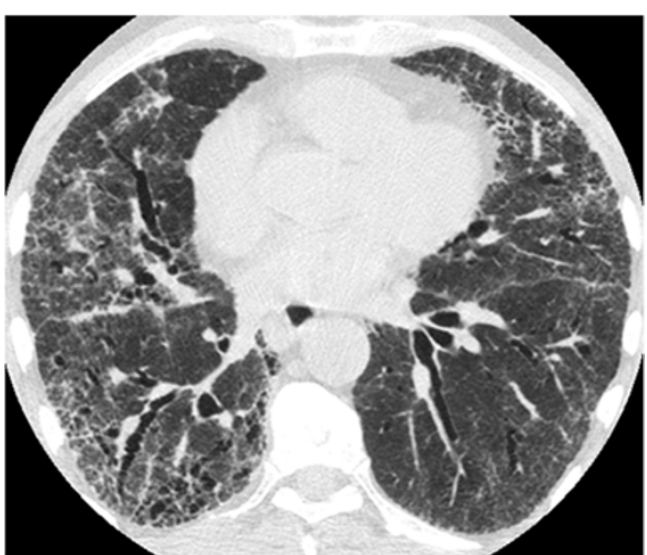

Fig. 5 Idiopathic pulmonary fibrosis. HRCT at the level of the lower lobes shows a subpleural "reticular pattern" characterised by thickened interlobular septae, thickened intralobular interstitium, traction bronchiolectasis and bronchiectasis and minimal honeycombing

Mimics The major mimics of idiopathic pulmonary fibrosis and therefore of reticular pattern are non-specific interstitial pneumonia (NSIP), asbestosis, chronic hypersensitivity pneumonitis, rheumatoid arthritis, desquamative interstitial pneumonia (DIP) and end-stage sarcoidosis.

NSIP is characterised by lower lobe and subpleural predominance of mild reticulation (depending on the subtype of NSIP, i.e. cellular, mixed, fibrotic), ground-glass opacity that may be pure, areas of consolidation and minimal if any honeycombing. HRCT may differentiate idiopathic pulmonary fibrosis (IPF) from NSIP in most cases, although considerable overlap may exist [15]. Clues that may differentiate NSIP over IPF are the presence of more ground-glass opacity and less - if any-honeycombing [16] (Fig. 6).

Asbestosis also presents with reticular pattern as the predominant pattern and may therefore mimic IPF. It is characterised by the presence of subpleural reticulation with subpleural dot-like opacities, at the early stages. There may be areas of ground-glass opacity and the findings are predominantly at the posterior and basal segments. At later stages honeycombing is a common finding. Clues for the differential diagnosis from IPF may be the presence of pleural plaques,

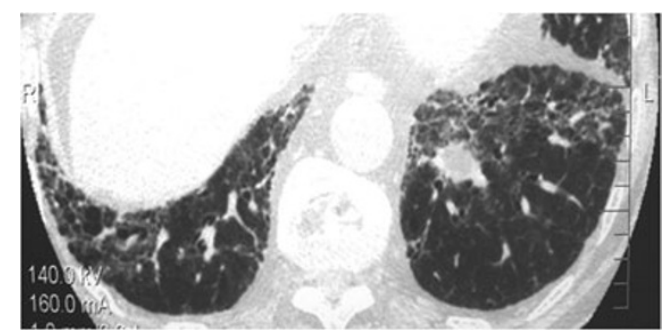

Fig. 6 Non-specific interstitial pneumonia. HRCT at the level of the lower lobes exhibits a "reticular pattern" characterised by the presence of thickened peribronchovascular interstitium and traction bronchiolectasis and bronchiectasis 
diffuse pleural thickening, subpleural lines or parenchymal bands [17] (Fig. 7).

Chronic hypersensitivity pneumonitis (HP) presents with patchy, peripheral or peribronchovascular reticulation and may mimic IPF. It is characterised by the presence of traction bronchiectasis and bronchiolectasis and minimal honeycombing. Areas of ground-glass opacity and hazy centrilobular micronodules may coexist. Relative sparing of the lung bases may be seen and there is no zonal predominance. Lung cysts may be noted in $40 \%$ of patients. The CT findings most helpful in differentiating chronic HP from idiopathic IPF and NSIP are the presence of lobular areas of decreased attenuation and vascularity, the presence of centrilobular nodules and the other than lower zone predominance. There is $50 \%$ diagnostic accuracy of HRCT in providing confident diagnosis between chronic HP, IPF and NSIP [18] (Fig. 8).

The most common histological types of interstitial lung disease in rheumatoid arthritis (RA) are UIP and NSIP and therefore RA may present with a reticular pattern mimicking entirely IPF or NSIP. The HRCT appearance is subpleural and basal reticulation with mild honeycombing. Differentiating findings may be the associated centrilobular nodules and frank bronchiectases reflecting coexistence of airways disease, as well as pleural thickening and pleural effusion [19] (Fig. 9).

Desquamative interstitial pneumonitis (DIP) may rarely mimic IPF, as it may present with subpleural basal minor reticulation. Honeycombing is usually absent, although in late stages minimal honeycombing may be seen. Groundglass opacity is a common finding and the presence of emphysema and lung cysts may help in the differential diagnosis [20] (Fig. 10).

Occasionally end-stage sarcoidosis presents with a pure reticular pattern mimicking IPF. It may be characterised by

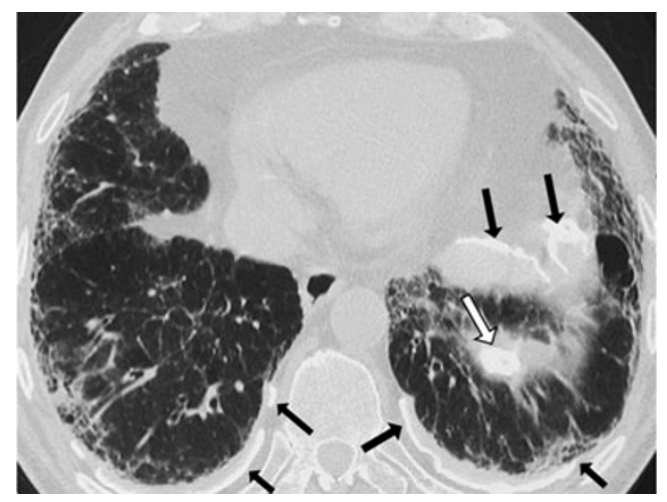

Fig. 7 Asbestosis. HRCT scan at the level of the lower lobes shows "reticular pattern" characterised by the presence of subpleural traction bronchiolectasis and bronchiectasis, honeycombing and thickening of the peribronchovascular interstitium and interlobular septae. The presence of calcified subpleural and diaphragmatic pleural plaques (white and black arrows) gives a definitive clue for the diagnosis of asbestosis

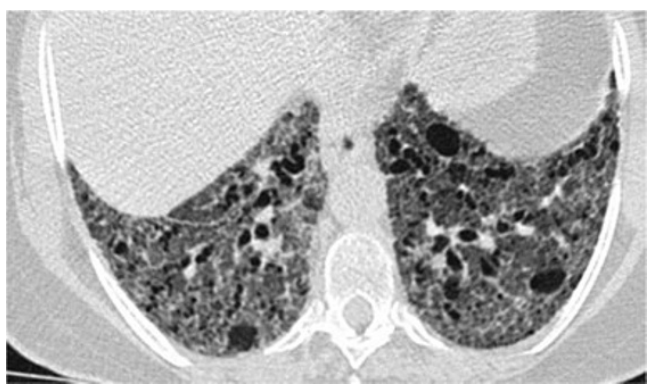

Fig. 8 Chronic hypersensitivity pneumonitis. HRCT at the level of the lower lobes demonstrates a coarse "reticular pattern" with traction bronchiolectasis and bronchiectasis, thickening of the intralobular and peribronchovascular interstitium and ground-glass opacity. The presence of lobular areas of decreased attenuation offers a "key" for the diagnosis of chronic hypersensitivity pneumonitis

thickening of interlobular septae, presence of intralobular linear opacities, traction bronchiectasis and honeycombing. Key findings that support the diagnosis of sarcoidosis are the middle-upper lung zone predominance, the nodular thickening of the peribronchovascular interstitium and the symmetric lymphnode enlargement, which may occasionally be calcified [7] (Fig. 11).

\section{Ground-glass pattern}

Ground-glass pattern on HRCT is defined as hazy increase in opacity with preservation of bronchial and vascular markings. Whatever the cause may be-i.e. the partial filling of airspaces, the interstitial thickening, the partial collapse of alveoli or the increased capillary blood volume - they all lead to partial displacement of air [4].

Classic entity The classic entity appearing with groundglass pattern as the predominant pattern is subacute hypersensitivity pneumonitis (HP) characterised by symmetric

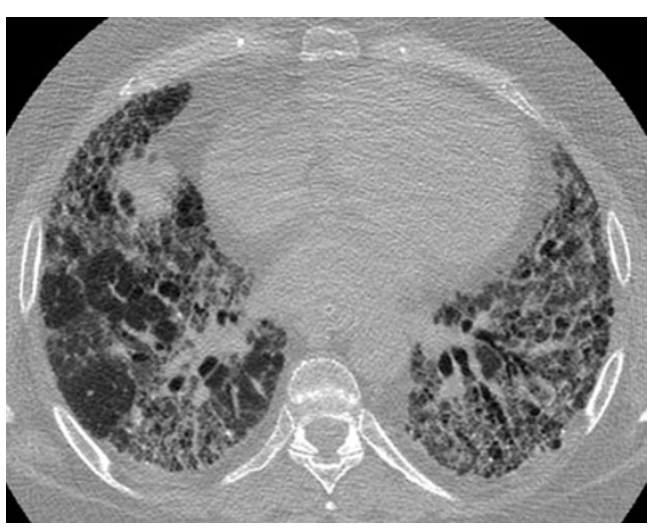

Fig. 9 Rheumatoid arthritis associated pulmonary fibrosis. HRCT at the level of the lower lobes exhibits a coarse "reticular pattern" consisted of traction bronchiolectasis and bronchiectasis, thickening of the intralobular and peribronchovascular interstitium, ground-glass opacity and honeycombing 


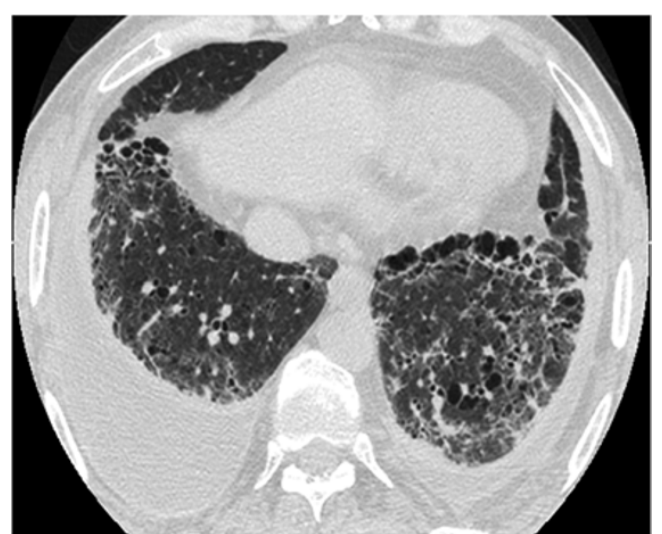

Fig. 10 Desquamative interstitial pneumonia (DIP). HRCT at the level of the lower lobes demonstrates a "reticular pattern" consisted of subpleural traction bronchiolectasis and bronchiectasis, thickening of the peribronchovascular interstitium, ground-glass opacity and minimal honeycombing. Bilateral pleural effusions - which are not a finding of DIP-are attributed to cardiac failure

patchy or diffuse bilateral areas of ground-glass opacity associated with poorly defined small centrilobular nodules. The above findings may be diffuse throughout the lungs or predominate in the middle and lower lung zones. A characteristic finding that helps differentiate subacute HP from other mimics is the presence of lobular areas of decreased attenuation and vascularity on inspiratory images and airtrapping on expiratory images [18] (Fig. 12). Another clue form the clinical history is the absence of smoking since HP is much less common in smokers [21].

Mimics The most common "mimics" of ground-glass pattern are respiratory bronchiolitis-associated interstitial lung disease (RB-ILD), desquamative interstitial pneumonia (DIP), and Pneumocystis pneumonia.

Respiratory bronchiolitis-associated interstitial lung disease (RB-ILD) may also demonstrate with ground-glass pattern as

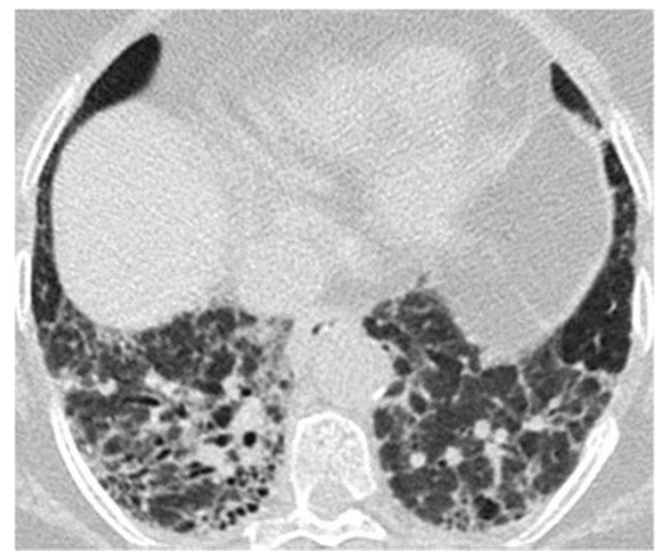

Fig. 11 End-stage sarcoidosis. HRCT at the level of the lower lobes demonstrates a coarse "reticular pattern" with architectural distortion, subpleural traction bronchiolectasis and bronchiectasis, thickening of the peribronchovascular interstitium and honeycombing

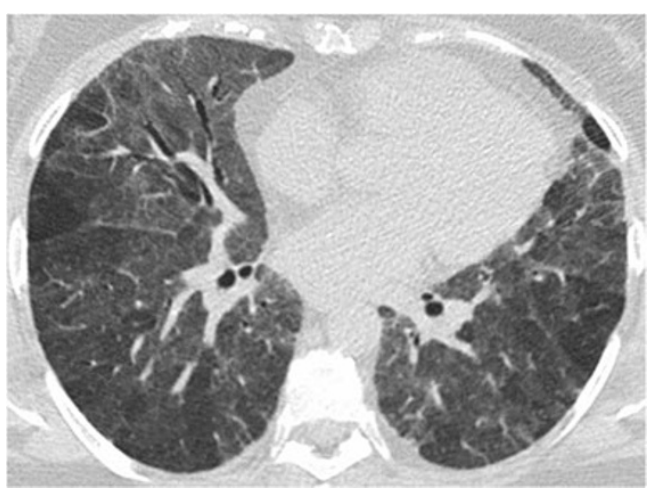

Fig. 12 Subacute hypersensitivity pneumonitis. HRCT shows geographic areas of "ground-glass pattern" with a few spared secondary lobules and no associated findings of fibrosis

the predominant pattern and mimic subacute hypersensitivity pneumonitis. RB-ILD may exhibit moderate to extensive bilateral ground-glass opacities associated with poorly defined centrilobular nodules of ground-glass attenuation [22] (Fig. 13). Air-trapping seen on end-expiratory CT and bronchial wall thickening are auxiliary findings. Occasionally, mild reticulation may be seen in the lung bases. Clues for the distinction between RBILD and subacute HP are the presence of mild emphysema in the upper lobes and the invariable history of smoking as HP is uncommon in smokers [21].

Desquamative interstitial pneumonia (DIP) typically manifests with diffuse ground-glass opacity as the primary pattern with a subpleural and lower lung predominance. A mild subpleural reticular pattern may be seen in half of the cases. Honeycombing and traction bronchiectasis are minimal-if any. Centrilobular emphysema is also seen in DIP as in RBILD, but mild lower zone reticulation is probably more often expected in DIP rather than RBILD [23]. History of smoking should accompany a diagnosis of DIP as it is almost invariably associated with cigarette smoking.

Pneumocystis pneumonia typically presents with extensive ground-glass attenuation that may be patchy or diffuse

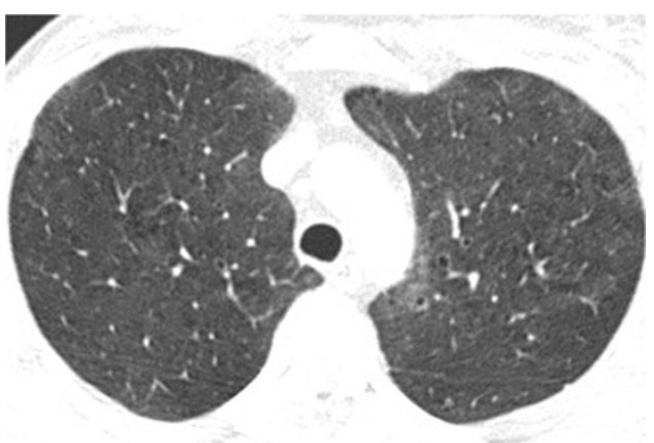

Fig. 13 Respiratory bronchiolitis associated interstitial lung disease. HRCT at the level of the upper lobes shows an almost diffuse "groundglass pattern" with small centrilobular radiolucent areas representing concomitant centrilobular emphysema 
with a central, perihilar and upper lobe predominance. Accompanying findings may be the thickening of the interlobular septae and rarely the "crazy paving" pattern. Less common manifestations may include the upper lobe lung cysts and areas of consolidation. Differentiating findings from HP may be the presence of upper lobe cysts and the associated "crazy paving" pattern [24] (Fig. 14). History of immunosuppression, and especially AIDS, favours the diagnosis of Pneumocystis pneumonia.

\section{Crazy paving pattern}

"Crazy paving" pattern is characterised on HRCT by the presence of thickened interlobular septae and intralobular lines superimposed on a background of ground-glass opacity, resembling irregularly shaped paving stones [4].

Classic entity This pattern was initially described in alveolar proteinosis, which typically presents bilateral groundglass opacities associated with smooth septal thickening in a patchy and geographic distribution [25] (Fig. 15). The findings are predominantly located at perihilar and lower lobe zones and the differential diagnosis from pulmonary oedema is based on the absence of cardiac enlargement and the lack of upper lobe vessels distention.

Mimics The most commonly encountered mimics of "crazy paving" pattern are Pneumocystis pneumonia, exogenous lipoid pneumonia, sarcoidosis, diffuse alveolar haemorrhage, viral and opportunistic infections and invasive mucinous adenocarcinoma.

Pneumocystis pneumonia may mimic alveolar proteinosis-although it may rarely present with "crazy paving" pattern as the predominant pattern, especially at the resolving or subacute stage [26]. Histological features contributing to the ground-glass attenuation are the foamy nature of the alveolar exudates and the thickening of the alveolar walls by

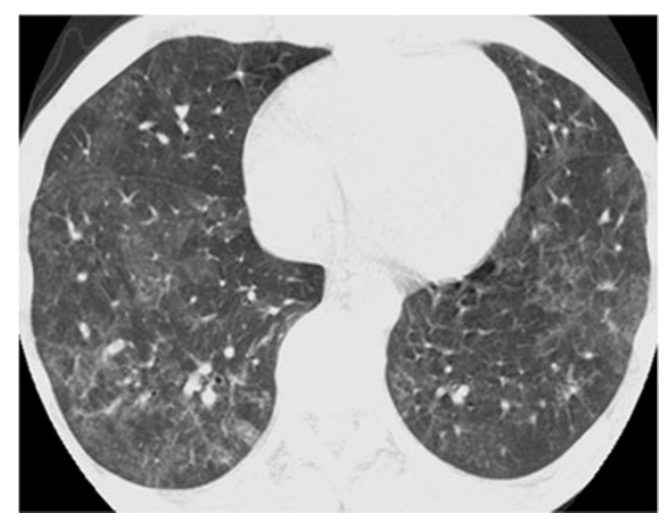

Fig. 14 Pneumocystis pneumonia. HRCT at the level of the lower lobes demonstrates geographic areas of "ground-glass pattern" with no associated findings of pulmonary fibrosis or lung cysts

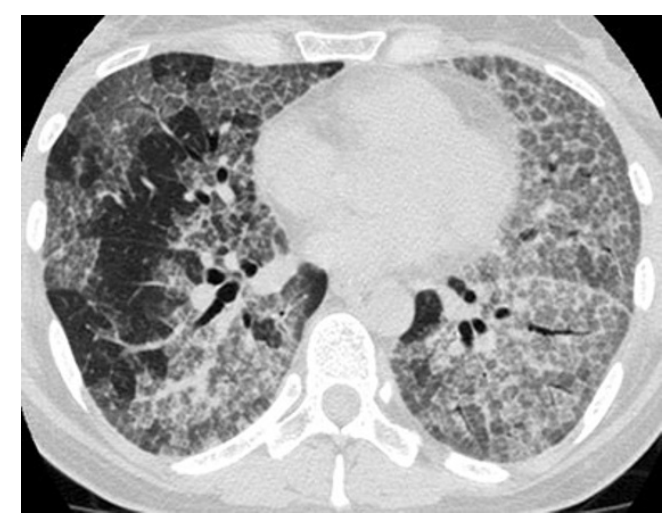

Fig. 15 Alveolar proteinosis. HRCT at the level of the lower and middle lobes demonstrates a "crazy paving pattern" characterised by geographic areas of involved pulmonary lobules with ground-glass opacity surrounded by thickened interlobular septae

oedema and cellular infiltrates [27]. Clues that are in favour of Pneumocystis pneumonia may be the coexistence of upper lobe lung cysts, and the knowledge of the stage of the disease during treatment.

Although exogenous lipoid pneumonia (ELP) typically presents with consolidation, that is typically low in attenuation $(-100 \mathrm{HU})$, it may also manifest with geographic areas of ground-glass attenuation surrounded by interlobular septal thickening, representing a "crazy paving" pattern. In terms of HRCT, distinctive features that favour lipoid pneumonia over alveolar proteinosis are the presence of ill-defined centrilobular nodules and the coexistence of consolidation [28]. Transbronchial biopsy, bronchoalveolar lavage and a history of oil ingestion are usually diagnostic for ELP.

Sarcoidosis may rarely present with a "crazy paving" pattern as the predominant pattern, but since sarcoidosis is the greatest mimicker of interstitial lung diseases, one should bear in mind this rare but significant "face" of sarcoidosis [27, 29] (Fig. 16). Key findings for the diagnosis of sarcoidosis are the more typical findings of sarcoidosis that may coexist as ancillary findings, i.e. the micronodules along all aspects of interstitium, the beading of the fissures and the lymphadenopathy.

Diffuse alveolar haemorrhage may result from various causes, including systemic, autoimmune, idiopathic and drug-induced reactions. Diffuse or focal pulmonary haemorrhage may manifest a "crazy paving" pattern especially at the subacute stage, representing the accumulation of hemosiderin-laden macrophages in the interstitium [27, 30] (Fig. 17). The association with areas of consolidation and the clinical history of haemoptysis are helpful for diagnosis.

Viral and opportunistic infections (adenovirus, herpes simplex, influenza virus, cytomegalovirus, respiratory syncytial virus and toxoplasmosis) in immunocompromised patients may commonly manifest with "crazy paving pattern", as the 


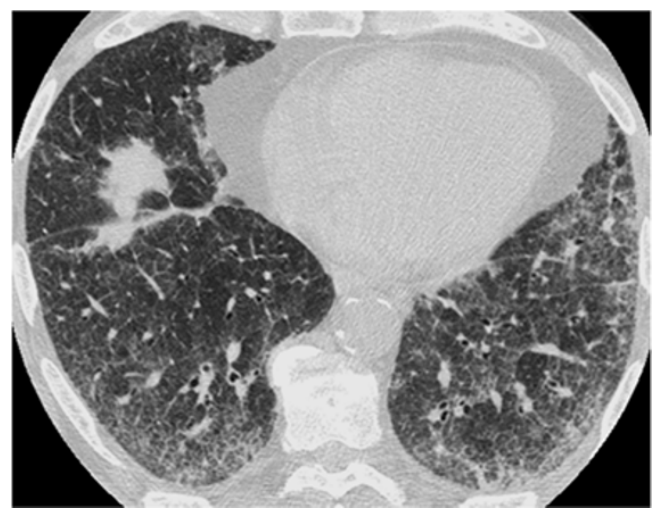

Fig. 16 Sarcoidosis. HRCT at the level of the lower lobes demonstrates a "crazy paving pattern" at the subpleural areas of the lungs associated with smoothly and nodular thickened interlobular septae. Note the "beaded" appearance of the major fissures, especially on the left

predominant pattern. Key finding in these cases is the knowledge of immunosuppression from the clinical history [31].

One of the many faces of adenocarcinoma on HRCT is "crazy paving", in which the ground-glass attenuation reflects low-density intra-alveolar material (glycoprotein) and the superimposed interlobular septal thickening, which is caused by interstitial infiltration from inflammatory or tumour cells. Key finding in the diagnosis of invasive mucinous adenocarcinoma is the non-resolving consolidation or ground-glass opacity on follow-up imaging [32] (Fig. 18).

\section{Mixed ground-glass-reticular pattern}

Occasionally, there may be coexistence of ground-glass pattern and reticular pattern - as defined earlier - in the same areas of the lungs and this combination is invariably equal to the presence of irreversible fibrosis [33].

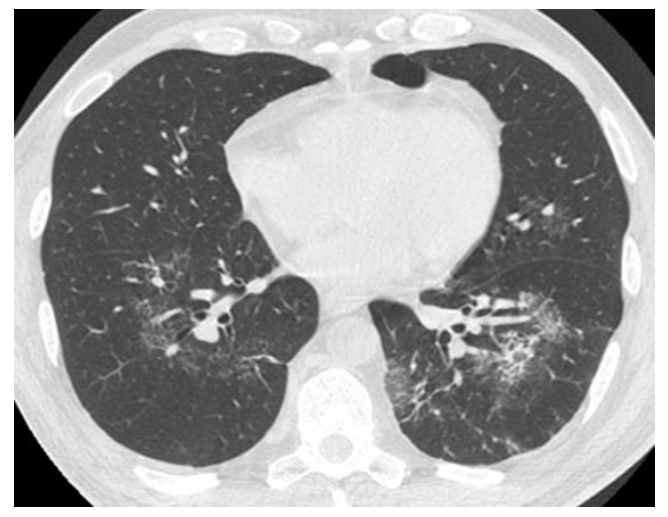

Fig. 17 Alveolar haemorrhage in a patient with history of haemoptysis 2 days before. HRCT at the level of the lower and middle lobes exhibits small geographic areas of "crazy paving pattern" (pulmonary lobules with ground-glass opacity surrounded by thickened interlobular septae) consistent with alveolar haemorrhage in remission

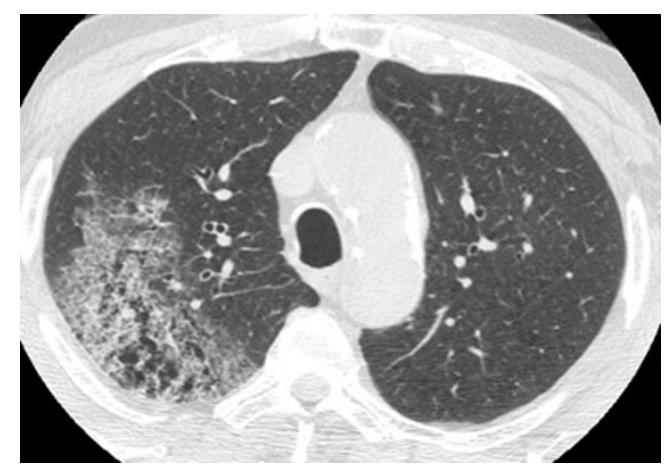

Fig. 18 Invasive mucinus adenicarcinoma. HRCT at the level of the upper lobes shows a focal area of "crazy paving pattern" in the right upper lobe intermingled with centrilobular emphysema

Classic entity Non-specific interstitial pneumonia (NSIP) presents most commonly with this combination of patterns as the predominant pattern. It manifests lower lobe predominant ground-glass opacities associated with mild reticulation. Distinctive findings are the absence or minimal honeycombing and the relative subpleural sparing of reticulation and ground-glass opacity [15, 16, 18] (Fig. 19).

Mimics The most clinically important mimics of mixed ground-glass-reticular pattern are scleroderma, idiopathic pulmonary fibrosis (IPF) and desquamative interstitial pneumonia (DIP).

Pulmonary involvement of scleroderma mimics NSIP as this is the most common histological type of fibrosis with which it presents [34]. Therefore, the two entities may be indistinguishable from each other in terms of HRCT findings regarding the interstitium. Key findings favouring scleroderma are the possible dilatation of the oesophagus and the dilatation of the pulmonary artery (exceeding the diameter of the adjacent ascending aorta) [35] (Fig. 20).

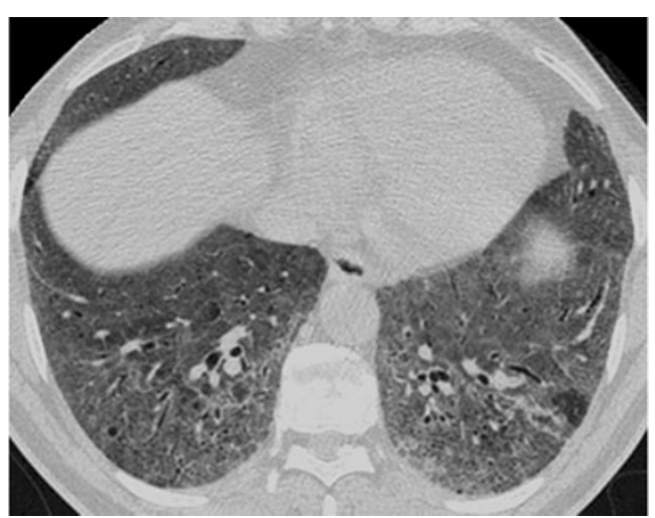

Fig. 19 Non-specific interstitial pneumonia. HRCT at the level of the lower lobes exhibits a mixed "ground-glass and reticular pattern" characterised by diffuse ground-glass opacity and traction bronchiolectasis. There is no associated honeycombing 


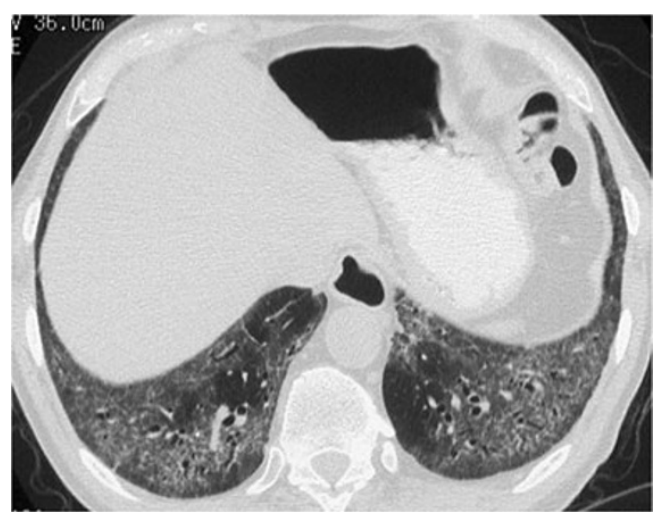

Fig. 20 Scleroderma. HRCT at the level of the lower lobes demonstrates a mixed "ground-glass and reticular pattern" characterised by geographic areas of ground-glass opacity and traction bronchiolectasis. Note the characteristic $5 \mathrm{~mm}$ subpleural sparing, which is characteristic for NSIP - the most common histological type of scleroderma associated pulmonary fibrosis

Idiopathic pulmonary fibrosis (IPF) may occasionally be indistinguishable from NSIP, as IPF may present less commonly with the combination of ground-glass and reticular pattern as the predominant pattern. HRCT has a specificity ranging from 63 to $70 \%$ in distinguishing IPF from NSIP [15] (Fig. 21). Differential diagnosis in favour of IPF is based on the presence of less ground-glass opacity and more coarse reticulation and honeycombing.

Although "pure" desquamative interstitial pneumonia $(D I P)$ is a rare entity, its most common pattern is that of ground-glass opacity associated with mild reticulation, which may mimic NSIP $[20,36]$ In DIP there is no subpleural sparing while coarse reticulation, such as honeycombing, is usually absent (Fig. 22). A minor CT feature which may help in the differential diagnosis is the presence of small microcysts that may either represent emphysema (since the patients are almost always smokers), bronchiolectasis or dilated alveolar ducts (without any obvious honeycomb fibrosis) [37].

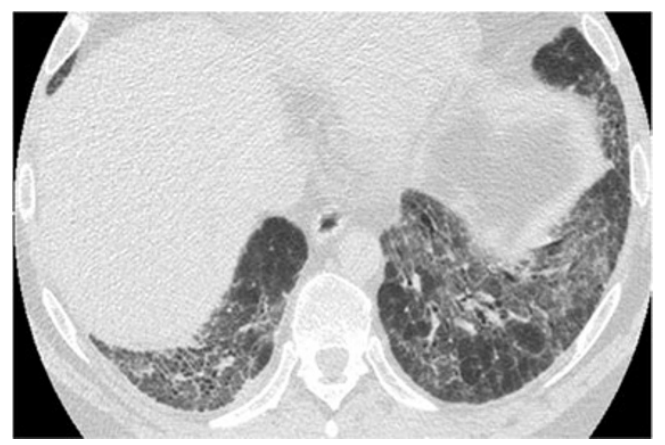

Fig. 21 Idiopathic pulmonary fibrosis. HRCT at the level of the lung bases exhibits a mixed "ground-glass and reticular pattern" characterised by geographic areas of ground-glass opacity within which traction bronchiolectasis and bronchiectasis is seen. Honeycombing is minimal, if any

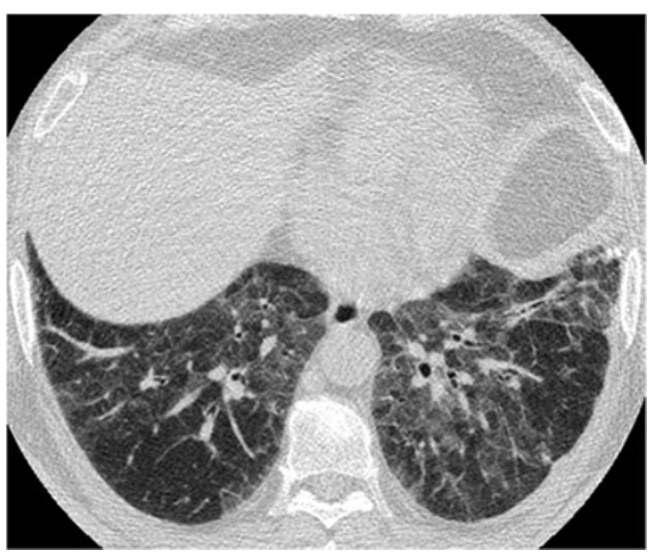

Fig. 22 Desquamative interstitial pneumonia. HRCT at the level of the lower lobes demonstrates geographic areas of mixed "ground-glass opacity and mild reticulation". Honeycombing is absent

\section{Nodular pattern}

A nodular pattern is characterised on HRCT by the presence of numerous rounded opacities that range from $2 \mathrm{~mm}$ to $1 \mathrm{~cm}$ in diameter, with micronodules defined as smaller than $3 \mathrm{~mm}$ in diameter [38]. The differential diagnosis - apart from the clinical setting and the ancillary findings - is 1 argely based on the three different patterns of anatomic distribution: perilymphatic, random, and centrilobular. The perilymphatic distribution is characterised by presence of nodules along the peribronchovascular interstitium, interlobular septa and subpleural areas. Random distribution demonstrates nodules without any particular site predominance. Centrilobular nodules are located several millimetres away from the pleural surfaces, interlobar fissures or interlobular septa. Centrilobular distribution further divides into nodules with and without tree-inbud distribution. Moreover centrilobular nodules without any "tree-in-bud" pattern may be either of homogeneous density or of ground-glass texture.

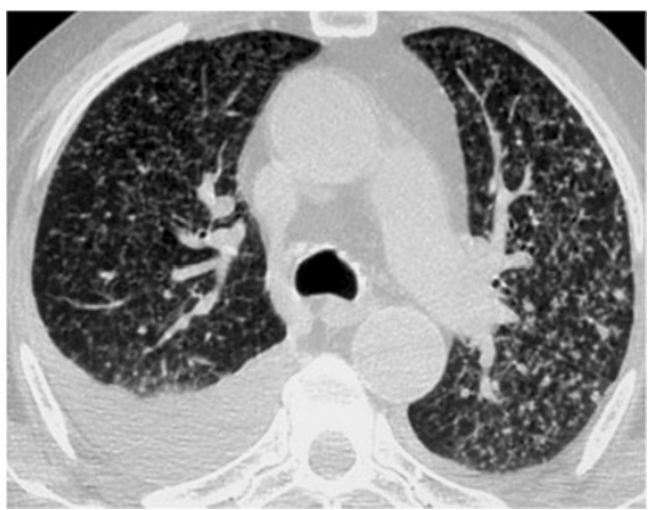

Fig. 23 Sarcoidosis. HRCT at the level of the upper lobes exhibits a "nodular without tree-in-bud pattern" characterised by the presence of perilymphatic micronodules around the interlobular septae, fissures, bronchovascular bundles and pleura 


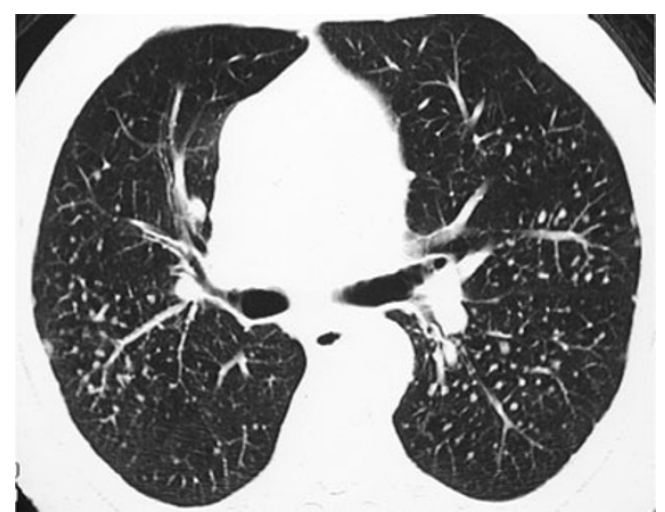

Fig. 24 Silicosis. CT at the level of the upper lobes exhibits a "nodular without tree-in-bud pattern" characterised by well-defined homogeneous centrilobular nodules that have an upper lobe and posterior predominance

\section{Nodular-perilymphatic}

Classic entity Sarcoidosis is typically characterised by a nodular pattern with perilymphatic distribution. A highly distinctive finding is the middle and upper lung zone predominance centrally at the perihilar region. Other findings that support the diagnosis of sarcoidosis are the beading of the fissures, the mediastinal and hilar lymphadenopathy that occasionally is calcified like "icing-sugar" and the presence of air-trapping on expiration [9] (Fig. 23).

Mimics Silicosis is a mimic of sarcoidosis and typically manifests with perilymphatic and centrilobular sharply defined micronodules ranging from 2 to $5 \mathrm{~mm}$. The nodules tend to be sharply defined and of homogeneous density and have a characteristic upper lobe and posterior predominance. Distinctive findings supporting the diagnosis of silicosis are the conglomerate masses formed by the coalescence of micronodules, the egg-shell calcification of lymph nodes (rare, only in $5 \%$ of cases) and the formation of pseudoplaques subpleurally, again by the coalescence of subpleurally based micronodules. Knowledge of history of exposure does not leave any doubt in most of the cases [39] (Fig. 24).

Although coal worker pneumoconiosis (CWP) and silicosis are two distinct entities caused by inhalation of different inorganic dusts, the radiographic and HRCT findings are often indistinguishable and the one disease mimics the other. A distinctive characteristic occasionally is that the micronodules of CWP tend to be less sharply defined and of more granular density than that of silicosis [40] (Fig. 25).

Lymphangitic carcinomatosis and lymphoproliferative diseases, such as lymphoma, may present with perilymphatic nodules but this does not usually constitute their predominant HRCT pattern and they are accompanied by

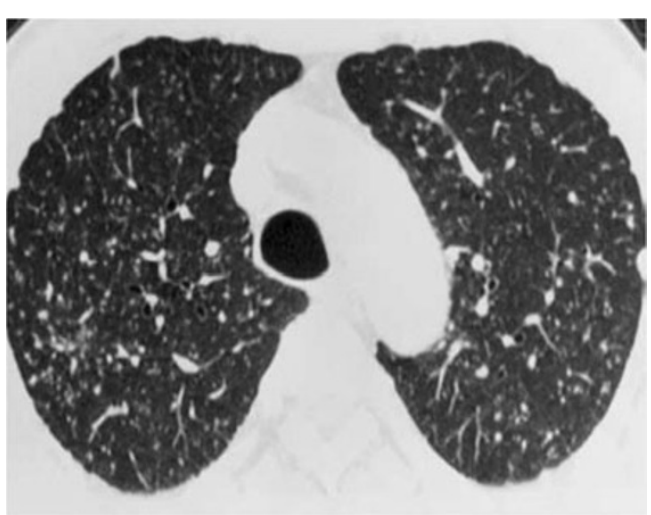

Fig. 25 Coal workers pneumoconiosis. HRCT at the level of the upper lobes exhibits a "nodular without tree-in-bud pattern" characterised by ill-defined centrilobular nodules of slightly variable size that have an upper lobe and posterior predominance

other findings such as septal thickening (in the former case) and large parenchymal nodules or masses (in the latter case) that help in the differential diagnosis [3, 41].

\section{Nodular-random}

Classic entity Miliary tuberculosis is a typical example of randomly distributed micronodules throughout both lungs ranging for 1 to $3 \mathrm{~mm}$. The micronodules are of uniform diameter and have an even distribution within the lungs. It may be impossible to distinguish infectious from neoplastic causes of miliary disease on imaging. However, miliary tuberculosis may more commonly be accompanied by lymphadenopathy and pleural effusion [42] (Fig. 26).

Mimics The most commonly encountered mimics of nodular pattern with random distribution are miliary metastatic disease, as well as other miliary infections such as miliary candidiasis [43].

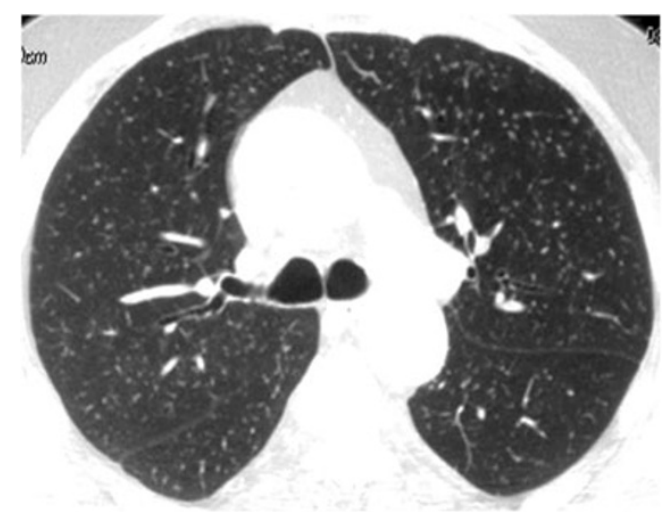

Fig. 26 Miliary TB. HRCT at the level of the upper lobes exhibits a "miliary nodular pattern" characterised by random micronodules diffusely and symmetrically distributed within the lungs having approximately the same size 
Miliary metastatic disease presents with randomly distributed nodules, which - unlike miliary tuberculosis - may have a variable size (up to $1 \mathrm{~cm}$ ), uniform or not. They are usually located in the outer third of the lung, have lower zone predominance and meet the criterion of "too many nodules to count" [41] (Fig. 27).

Nodular — centrilobular with tree-in-bud pattern

The tree-in-bud pattern represents centrilobular branching structures that resemble a budding tree. The pattern reflects a spectrum of endobronchiolar and peribronchiolar disorders, including mucoid impaction, inflammation, fibrosis and occasionally endovascular disorders such as neoplastic processes.

Classic entity This pattern was initially described for panbronchiolitis, which is a rare entity affecting mainly Japanese and Korean middle-aged men. It is characterised by diffuse bilateral distribution of "tree-in-bud" pattern with lower lobe predominance and associated bronchiolectasis and bronchiectasis, late in the disease process [44, 45] (Fig. 28). Distinctive findings from other causes of "treein-bud" pattern may be the diffuse distribution and the prominent air-trapping on expiration.

Mimics The most clinically important mimics of nodular with centrilobular tree-in-bud pattern are endobronchial spread of tuberculosis, atypical mycobacteria infection and metastatic tumour emboli.

Endobronchial spread of tuberculosis is a famous representative of this HRCT pattern, which can be differentiated from panbronchiolitis based on the more focal and asymmetric distribution of "tree-in-bud" pattern. Associated cavitated nodules, mediastinal lymphadenopathy and pleural effusions also favour the diagnosis of tuberculosis [46] (Fig. 29).

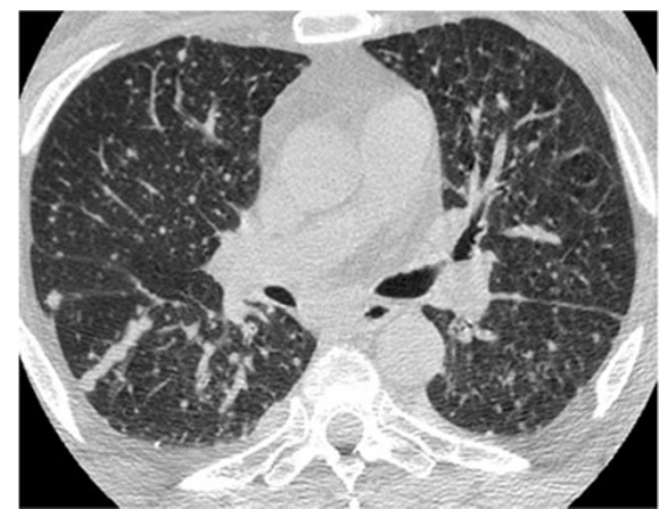

Fig. 27 Miliary metastatic disease. HRCT at the level of the upper lobes shows a "milary nodular pattern" characterised by random and perilymphatic micronodules diffusely distributed throughout the lungs that have a more variable size compared with Fig. 26

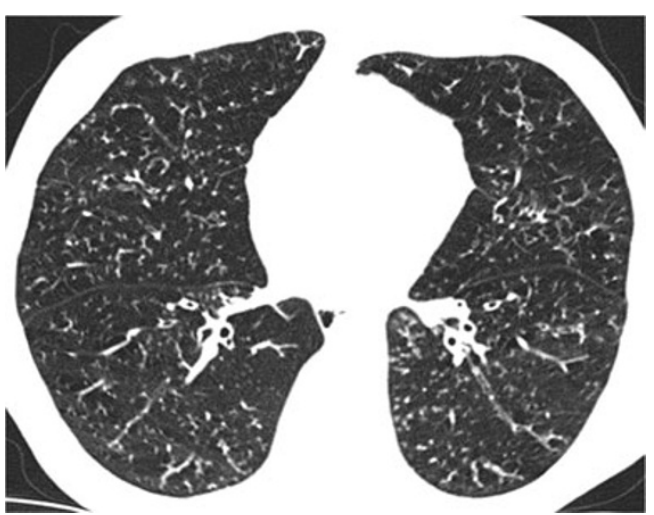

Fig. 28 Panbronchiolitis. HRCT at the level of the lower and middle lobes exhibits a "nodular with tree-in-bud pattern" diffusely throughout the lungs

Atypical mycobacteria infection may mimic endobronchial spread of tuberculosis presenting with scattered areas of "tree-in-bud" pattern. Key findings that favour the diagnosis of atypical mycobacteria infection are the presence of multilobar, cylindrical bronchiectasis that tends to be more severe in middle lobe and lingula and the characteristic age of the affected patients: elderly women [47, 48] (Fig. 30). Other less common causes of infection that may present with a nodular tree-in bud pattern are pulmonary candidiasis [49]. bronchoinvasive aspergillosis [50] and CMV pneumonia [51].

Metastastatic tumour emboli in pulmonary arterioles may also present with a "tree-in-bud" pattern, which in this case refers to the vascular rather than the bronchial tree. It is either caused by the filling of the centrilobular arteries with tumour emboli or by the fibrocellular intimal hyperplasia induced by tumour microemboli. Ancillary findings may be the multifocal dilatation and beading of arteries, the thickening of the interlobular septae and small wedge-shaped peripheral opacities, secondary to microinfarcts [52].

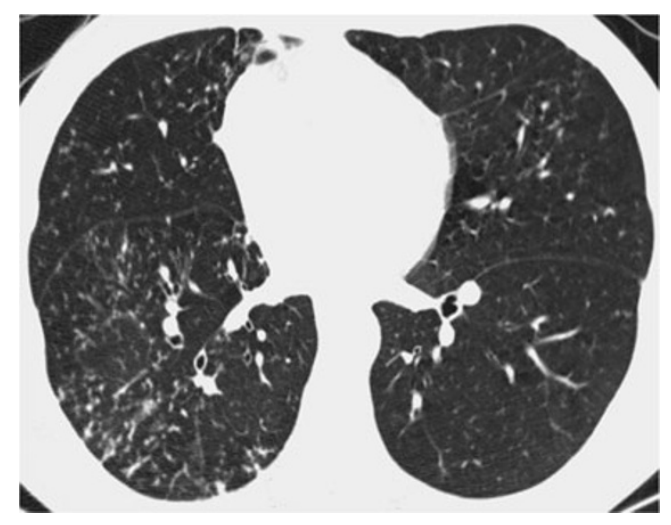

Fig. 29 Tuberculosis. HRCT at the level of the lower and middle lobes exhibits a "nodular with tree-in-bud pattern" predominantly in the right lower lobe, consistent with endobronchial spread of tuberculosis 


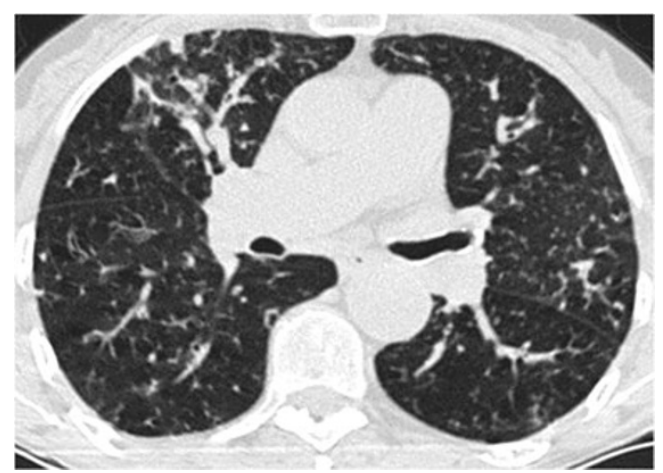

Fig. 30 Atypical mycobacteria infection in an elderly woman. HRCT at the level of the middle lobe, lingula and the superior segments of the lower lobes reveals a "nodular with tree-in-bud pattern" associated with mild cylindrical bronchiectasis in middle lobe and lingula and mucus plugging

Nodular-ill-defined centrilobular (without tree-in-bud) pattern

This pattern is characterised by the presence of poorly defined centrilobular nodules of ground-glass attenuation that appear from the pleural surface, fissures or interlobular septa by several millimetres $[4,41]$.

Classic entity The classic example for this pattern is subacute hypersensitivity pneumonitis, which commonly presents with this pattern as the predominant one (Fig. 31). Associated key findings are the geographic areas of ground-glass opacity and the lobular areas of decreased attenuation and vascularity with air-trapping on expiration. The centrilobular nodules are usually diffuse with middle \& lower lobe predominance. Apart from the integration with the clinical history of exposure and the significant improvement with the removal of the patient from the offending environmental agent, another helpful clue is the absence of smoking history since it is known that smokers are somehow "protected" from developing hypersensitivity pneumonitis $[53,54]$.

Mimics The most commonly encountered mimics of nodular ill-defined centrilobular pattern are respiratory bronchiolitis-associated with interstitial lung disease (RBILD), cryptogenic organising pneumonia, lymphocytic interstitial pneumonia and follicular bronchiolitis.

RBILD mimics hypersensitivity pneumonitis and may present with ill-defined centrilobular micronodules of ground-glass attenuation as the predominant pattern. In RB-ILD the micronodules display a distinctly upper lobe predominance and may be fewer in number than those seen in patients with subacute hypersensitivity pneumonitis [41] (Fig. 32). In RB-ILD there is centrilobular or paraseptal emphysema in the upper lobes, which is of mild severity despite the fact that most of the patients are heavy smokers.

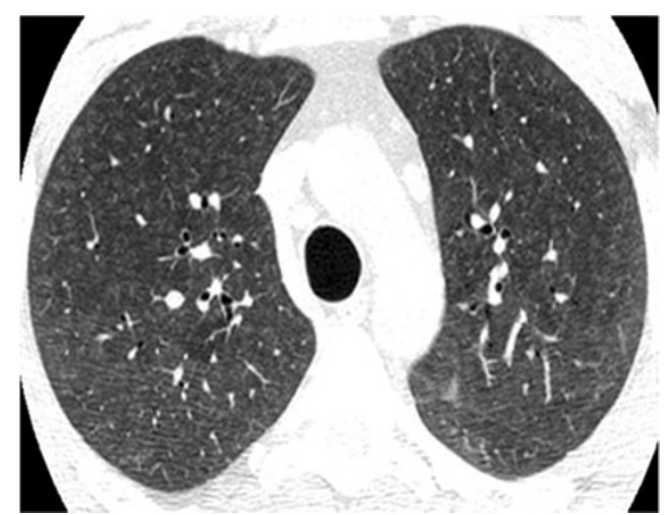

Fig. 31 Hypersensitivity pneumonitis. HRCT at the level of the upper lobes reveals an "ill-defined centrilobular nodular pattern" characterised by micronodules of ground-glass opacity that are diffusely distributed characteristically in the centre of the pulmonary lobules

The differential diagnosis between RBILD and HP is often impossible on imaging grounds only, and the distinction is based on the smoking history as hypersensitivity pneumonitis is uncommon in smokers [22, 55].

Cryptogenic organising pneumonia may rarely present with diffusely distributed, ill-defined centrilobular micronodules of less than $4 \mathrm{~mm}$ as the predominant pattern and thus mimic subacute hypersensitivity pneumonitis [56]. Organising pneumonia may occasionally present with a distinct bronchiolocentric distribution limited to the alveoli immediately adjacent to the involved bronchioles, thus giving a miliary pattern [57]. Distinction from hypersensitivity pneumonitis and RBILD is based on the absence of lobular areas of decreased attenuation and absence of emphysema (Fig. 33).

Lymphocytic interstitial pneumonia (LIP) and follicular bronchiolitis may both present with centrilobular micronodules of ground-glass attenuation as the predominant pattern and mimic subacute hypersensitivity pneumonitis. They are

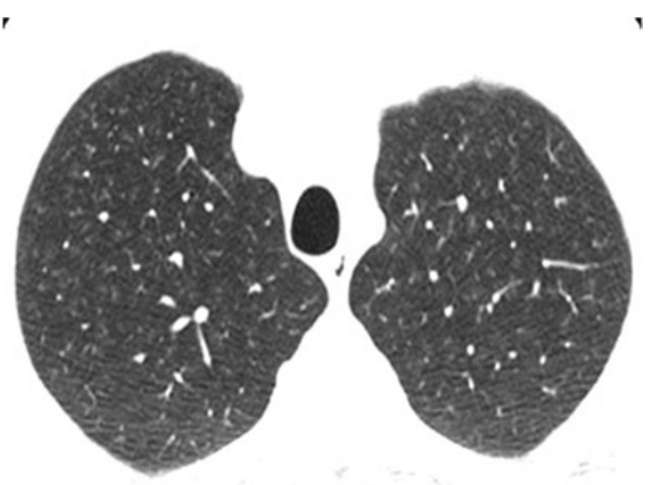

Fig. 32 RBILD. HRCT at the level of the upper lobes exhibits an "illdefined centrilobular nodular pattern" characterised by micronodules of ground-glass opacity that are diffusely distributed characteristically in the centre of the pulmonary lobules. In this case the history of smoking favours the diagnosis of respiratory bronchiolitis interstitial lung disease 


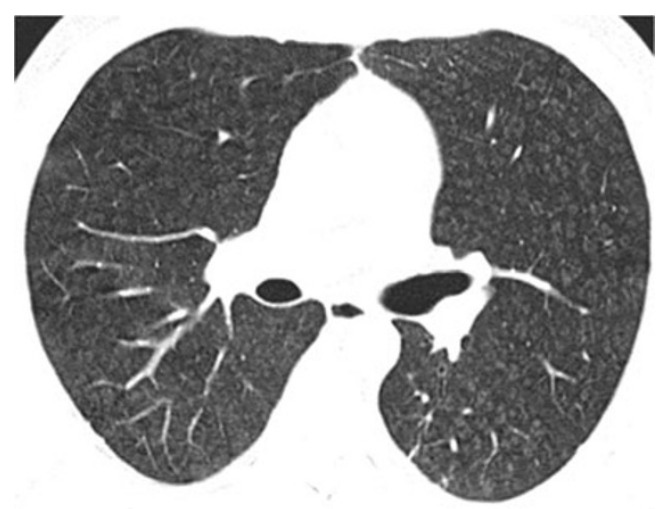

Fig. 33 COP. HRCT at the level of the upper lobes exhibits an "illdefined centrilobular nodular pattern" characterised by micronodules of ground-glass opacity that are diffusely distributed characteristically in the centre of the pulmonary lobules. The HRCT findings are almost indistinguishable from those in Fig. 31 or 32

considered to represent two ends of a spectrum, with follicular bronchiolitis localised more to the peribronchiolar region and with LIP being more diffuse in the secondary pulmonary lobule. In case of LIP distinctive findings on HRCT may be the associated thickening of the interlobular septae, thin-walled lung cysts, areas of ground-glass opacity and slightly enlarged mediastinal lymphadenopathy. A significant clue from the clinical history is that both entities are strongly associated with underlying autoimmune disease or immunodeficiency, Sjogren's syndrome, dysproteinaemia or AIDS [58, 59].

\section{Reticulonodular pattern}

A reticulonodular pattern is characterised by the co-occurrence of reticular and micronodular patterns. The micronodules may either be located at the centre of the reticular elements (centrilobular micronodules) or on the linear opacities representing septal or peribronchovascular micronodules [4].

Classic entity A typical example of predominantly reticulonodular pattern is sarcoidosis. It is characterised by the presence of perilymphatic and peribronchovascular micronodules, as well as of the thickening of interlobular septae and peribronchovascular interstitium and presence of intralobular linear opacities. The characteristic middle and upper lung zone distribution with central-parahilar predominance and the presence of mediastinal and hilar lymphadenopathy usually lead to the correct diagnosis, obviating the need for lung biopsy. Air-trapping on expiration is another key finding that usually accompanies sarcoidosis $[7,60]$ (Fig. 34).

Mimics Berylliosis is the most important mimic of sarcoidosis on imaging and cannot be distinguished from it even on histological basis. Its main HRCT manifestations are the co-

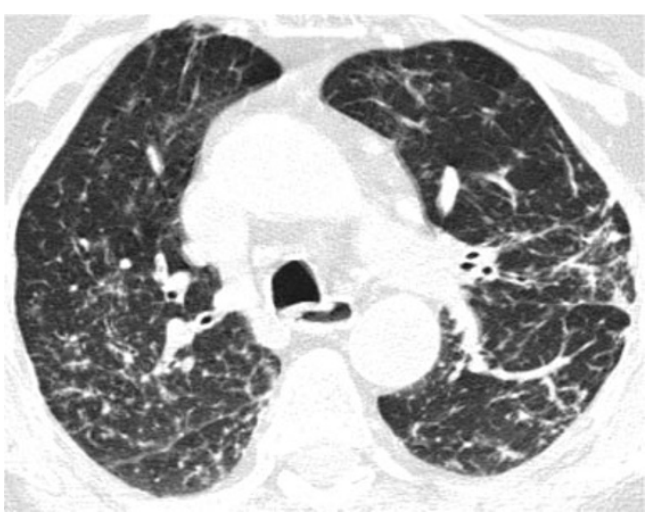

Fig. 34 Sarcoidosis. HRCT at the level of the upper lobes exhibits a "reticulonodular pattern" characterised by the presence of thickening of the interlobular septae and bronchovascular bundles, perilymphatic and perifissural micronodules and architectural distortion

occurrence of perilymphatic nodules, thickening of interlobular septa and peribronchovascular interstitial thickening [61]. Lymphadenopathy is expected to be less pronounced than sarcoidosis and the main key finding to reach the correct diagnosis seem to rest on the knowledge of history of exposure.

Lymphangitic carcinomatosis may occasionally present with a reticulonodular pattern characterised by irregular, nodular thickening of the interlobular septae and of the bronchovascular bundles. Differentiating CT findings from sarcoidosis are the preservation of the architecture of the secondary pulmonary lobule, the more diffuse distribution if it is secondary to extrapulmonary cancer or the confinement to one lobe or lung if it is secondary to lung cancer and the less common incidence of mediastinal lymphadenopathy [6].

\section{Cystic pattern}

Cystic pattern is composed by well-defined, round and circumscribed air-containing parenchymal spaces with a well-defined wall and interface with normal lung. The wall

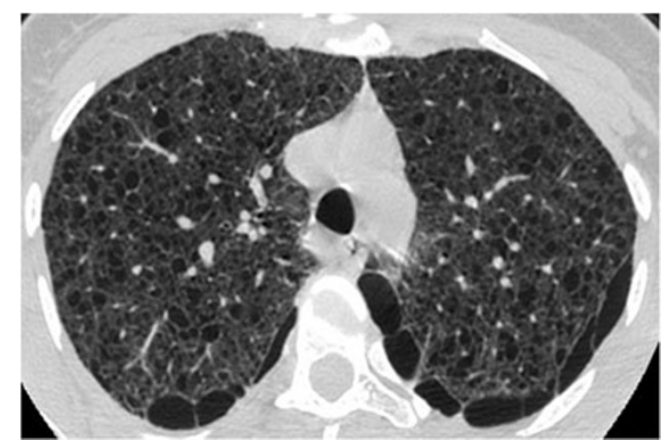

Fig. 35 LAM. HRCT at the level of the upper lobes exhibits a "cystic pattern" characterised by the presence of numerous thin walled "true" cysts of variable sized that are located in the lung parenchyma and paraseptally 


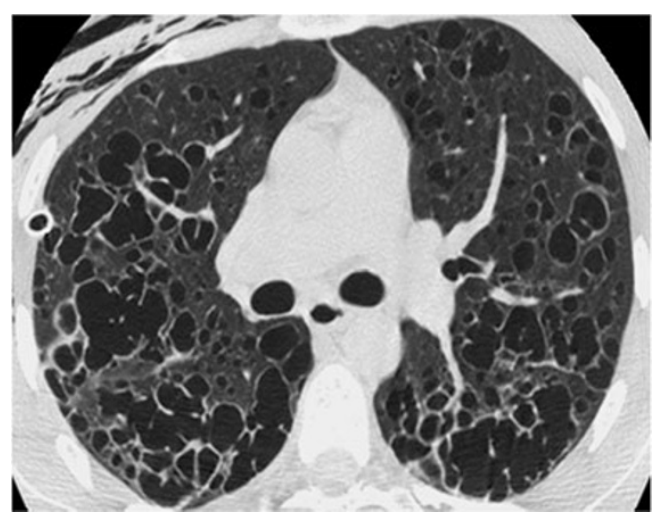

Fig. 36 Histiocytosis. HRCT at the level of the upper lobes reveals a "cystic pattern" characterised by numerous thin- and thick-walled "true" cysts with bizarre shapes and variable size. A drainage catheter is noted in the right pleural space in order to treat pneumothorax, which was the presenting symptom of this 27 -year-old heavy smoker

of the cysts may be uniform or varied in thickness, but usually is thin $(<2 \mathrm{~mm})$ and occurs without associated emphysema [4].

A classic entity presenting with cystic pattern is lymphangioleiomyomatosis (LAM), characterised by the presence of usually round, thin walled lung cysts which have no zonal predominance and are distributed diffusely including the costophrenic angles and the lung bases (Fig. 35). LAM may present with pleural effusion representing chylothorax. Key findings in terms of HRCT criteria is the diffuse distribution of cysts and in terms of clinical history the knowledge that it affects almost exclusively women in child bearing age [62].

Mimics The most clinically important mimics of cystic pattern are pulmonary histiocytosis, lymphocytic interstitial pneumonia (LIP) and centrilobular emphysema.

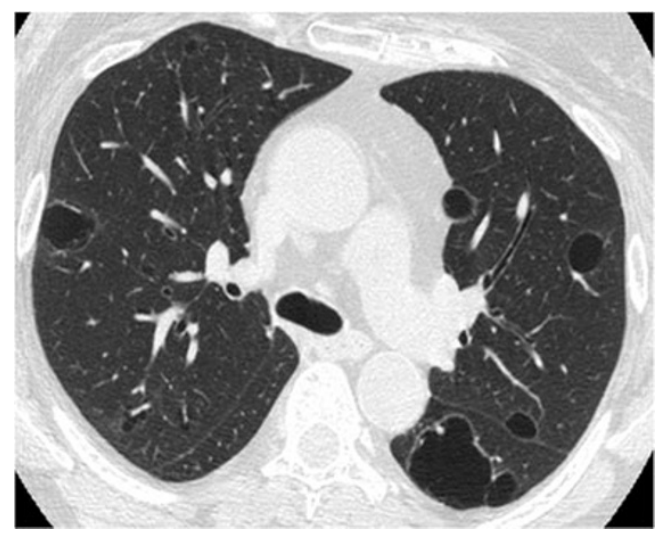

Fig. 37 Lymphocytic interstitial pneumonia. HRCT at the level of the upper lobes reveals a "cystic pattern" characterised by few thin-walled "true" cysts that may have rounded or more lobulated shape. The history of immunosuppression (AIDS, Sjogren, autoimmune disorders, dysproteinemia) favours the specific diagnosis of LIP

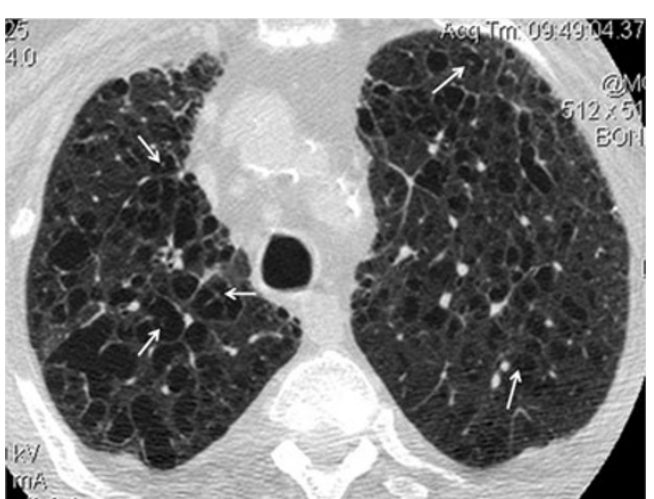

Fig. 38 Centrilobular emphysema. HRCT shows centrilobular areas of radiolucency with no discernible walls in most of the cases (except in those areas where there is thickening of the interlobular septae) and with the presence of a central white dot (at the centre of the radiolucencies) representing the centrilobular artery (white arrows). Centrilobular emphysema mimics occasionally interstitial diseases that present with a "true cystic pattern"

Pulmonary histiocytosis mimics LAM when it is in the purely cystic phase (third phase following the purely nodular and nodular-cystic phase). It is characterised by the presence of thin- and thick-walled lung cysts that have a bizarre shape, resembling cloverleaf. Cysts have a characteristic upper and middle lung zone predominance sparing the costophrenic angles and the lung bases (Fig. 36). Occasionally, few scattered nodules may coexist (representing Langerhans granulomas) as well as areas of ground-glass opacity. Key findings are the sparing of costophrenic angles and of the medial parts of anterior parts of middle lobe and lingula, the concurrence of even one nodule and the history of smoking since it occurs in young heavy smokers [23].

LIP may rarely present with diffuse lung cysts as the predominant HRCT pattern [63] (Fig. 37). Key findings in the differential diagnosis that favour LIP is the association with other ancillary findings such as diffuse or patchy areas of ground-glass opacity, ill-defined centrilobular nodules and thickening of the bronchovascular bundles. Moreover,

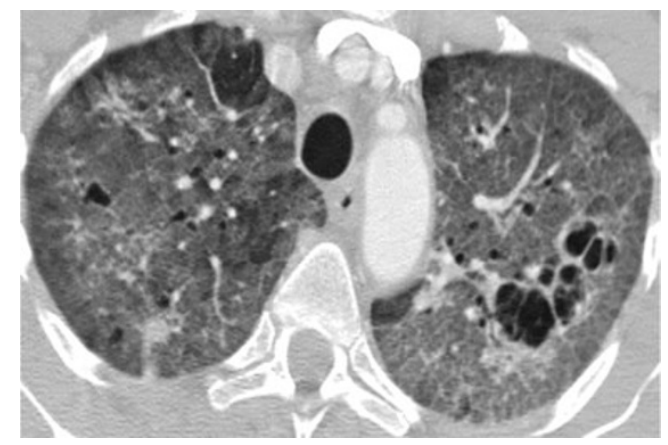

Fig. 39 Pneumocystis pneumonia in an AIDS patient. HRCT at the level of the upper lobes reveals a mixed "ground-glass and cystic pattern" characterised by the presence of diffuse areas of groundglass opacity and a few thin-walled multilocular cysts 


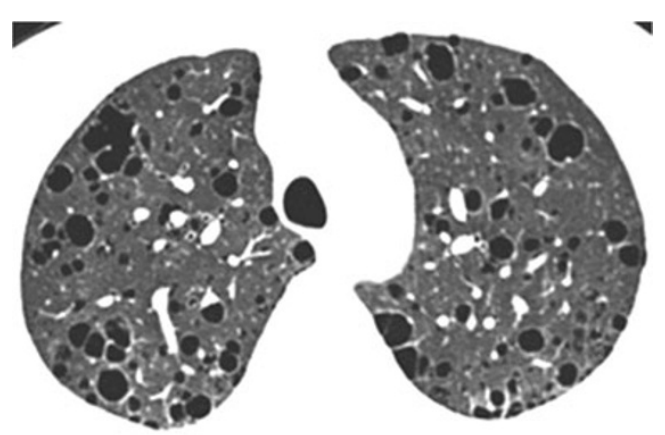

Fig. 40 Lymphocytic interstitial pneumonia in a female patient with Sjogren's disease. HRCT at the level of the upper lobes exhibits a mixed "ground-glass and cystic pattern" characterised by the presence of diffuse areas of ground-glass opacity and numerous thin-walled rounded and lobulated cysts of variable size

the clinical history of the primary disease causing some degree of immunosuppression (such as Sjogren's disease, AIDS, autoimmune disorders, dysproteinemia) gives a strong clue towards LIP.

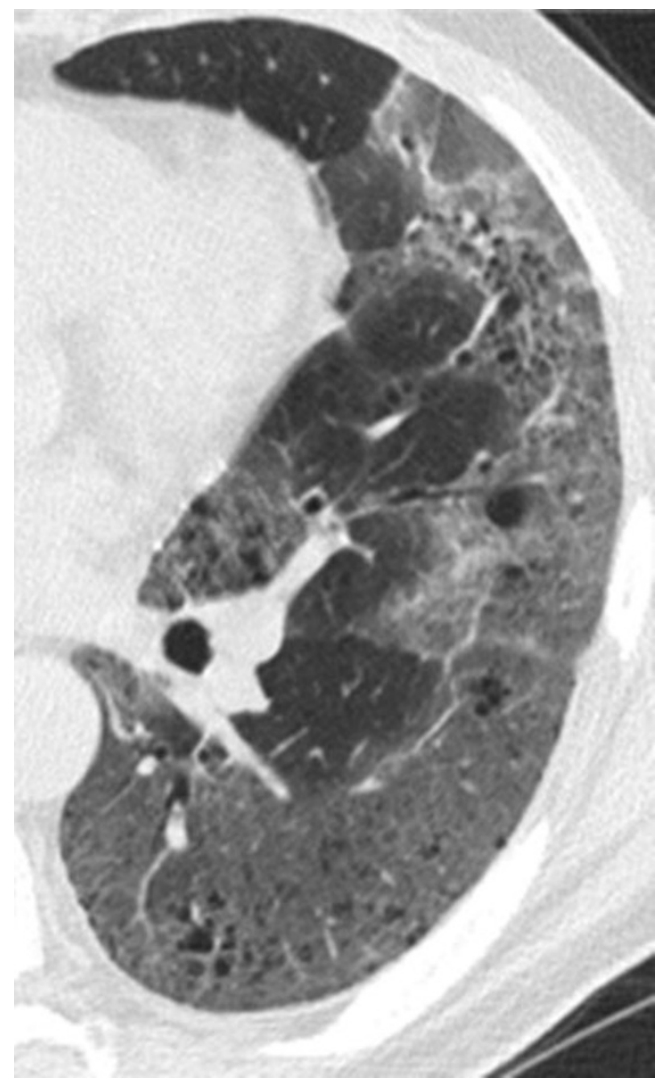

Fig. 41 Desquamative interstitial pneumonia. HRCT at the level of the left lower lobe (magnified view of the left lower lobe) reveals a mixed "ground glass and cystic pattern" characterized by the presence of patchy areas of ground glass opacity and numerous small scattered "lucencies" that may represent either true cysts, bronciolectasis or emphysema

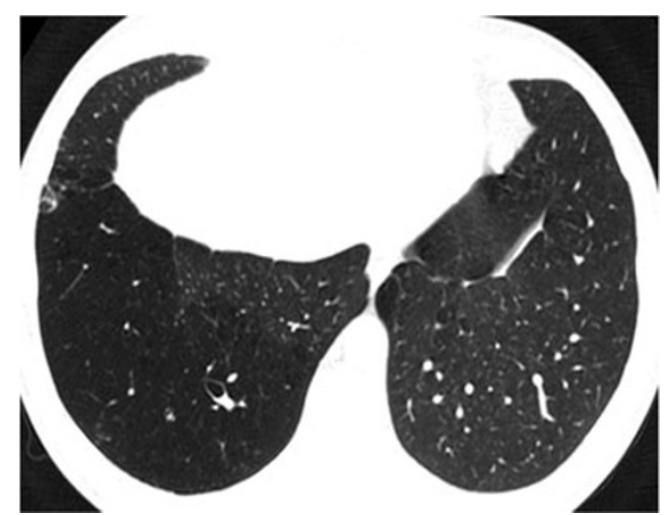

Fig. 42 Obliterative bronchiolitis. HRCT at the level of the lower lobes exhibits a "decreased attenuation pattern" characterised by patchy "black" areas with paucity of vessels and few scattered bronchiectasis

Centrilobular emphysema, although not characterised by the presence of "true" cysts, may occasionally mimic other interstitial lung diseases expressed by a cystic pattern. Distinctive findings on HRCT that favour centrilobular emphysema are the absence of perceptible wall of the small centrilobular lucencies with characteristic upper lobe predominance and the presence of a "white dot" in the middle of these lucencies representing the centrilobular artery [64] (Fig. 38).

\section{Combined cystic and ground-glass pattern}

This combined HRCT pattern is characterised by the cooccurrence of two already mentioned HRCT patterns, i.e. the cystic pattern and the ground-glass pattern.

Classic entity Classic example of this combined pattern as the predominant pattern is Pneumocystis pneumonia, which is characterised by the presence of patchy or diffuse bilateral ground-glass opacities with thick or thin-walled, irregular,

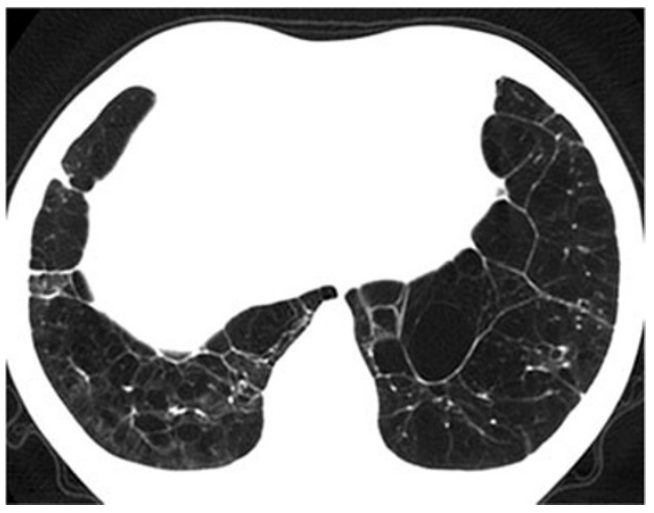

Fig. 43 Panlobular emphysema in a $\alpha-1$ antitrypsin deficiency patient. HRCT at the level of the lower lobes reveals a "decreased attenuation pattern" characterised by the presence of confluent "black pulmonary lobules surrounded by few interlobular septae resembling a "spider's web" 
Fig. 44 Obliterative

bronchiolitis in a patient with cystic fibrosis. HRCT at the level of the carina at (a) inspiration and (b) expiration reveals at expiration a "mosaic attenuation pattern" secondary to air-trapping (b) which is not revealed on inspiration (a)
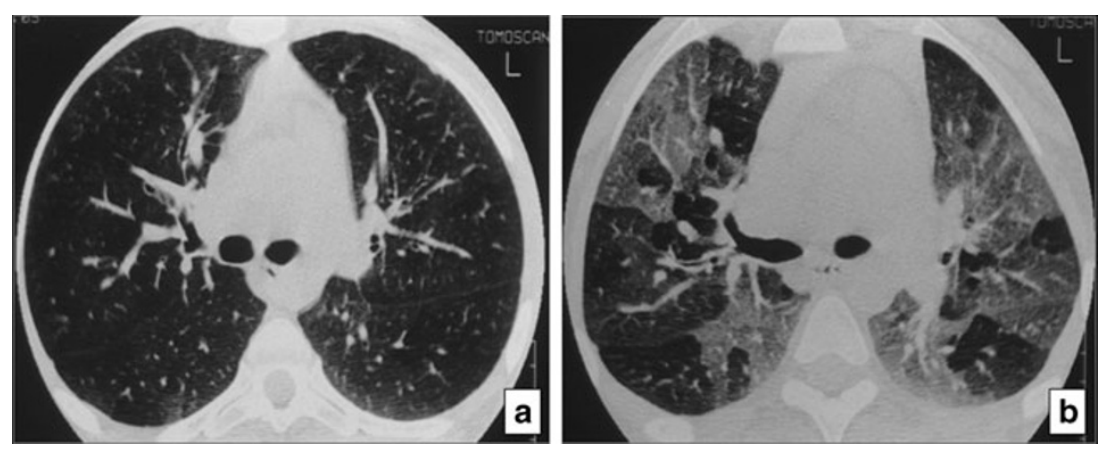

septated cysts in the upper lobes. A significant key from the clinical history is the history of immunosuppression or more specifically the history of AIDS [65] (Fig. 39).

Mimics The most clinically important mimics of combined cystic and ground-glass pattern are lymphocytic interstitial pneumonia, subacute hypersensitivity pneumonitis and desquamative interstitial pneumonia.

Lymphocytic interstitial pneumonia may present with this combined pattern as the predominant pattern and may occasionally mimic Pneumocystis pneumonia. Distinctive HRCT findings are the thin wall and the more rounded shape of the cysts, the possible associated thickening of the bronchovascular bundles and the mediastinal lymphadenopathy which occurs in $70 \%$ of these patients [58] (Fig. 40). Once again, the knowledge of the primary disease from the clinical history causing some degree of immunosuppression (such as Sjogren's disease, AIDS, Castleman's, AIDS, autoimmune disorders or dysproteinemia) gives a strong clue towards LIP.

Subacute hypersensitivity pneumonitis (HP) may also manifest with this combined HRCT pattern as the predominant pattern. However, in case of HP the lung cysts are reported to be few in number and thin-walled [66]. The distinctive HRCT finding is characteristic lobular areas of decreased attenuation, even from the inspiratory images and the absence of smoking history, since smokers very rarely develop HP [54].

Desquamative interstitial pneumonia (DIP) is another entity that may present with this combined pattern. In case of DIP, the diffuse or patchy areas of ground-glass opacity have lower lobe predominance and the scattered "lucencies" may or may not have true walls, either representing "true cysts" or else emphysema or traction bronchiectasis. The patient has to be a smoker based on the clinical history, since DIP develops almost always in heavy smokers [20, 36] (Fig. 41).

\section{Decreased attenuation pattern}

The decreased attenuation pattern is defined as areas of low density corresponding to parenchymal destruction and reduced perfusion, and attenuation of the pulmonary vasculature. It is also known as the "black lung" pattern.
Classic entity The classic example of this pattern is obliterative bronchiolitis, characterised by the presence of diffuse areas of low density with small calibre vessels seen within (vessel paucity). There is no zonal predominance and the distribution may be diffuse or patchy (see also "mosaic attenuation pattern"). Air trapping is noted on expiratory phase and there is also associated cylindrical bronchiectasis [67] (Fig. 42).

Mimics Panlobular emphysema is actually the only mimic of obliterative bronchiolitis and presenting as "lucent" lung with small or absent vessels and associated bronchial dilatation with bronchial wall thickening. HRCT features favouring panlobular emphysema are the typical lower lobe predominance, the characteristic presence of thick, "long" interlobular septae in lucent lung like a "web", the parenchymal destruction and the vascular distortion (Fig. 43). The clinical history of $\alpha-1$ antitrypsin deficiency undoubtedly leads to the correct diagnosis [68].

\section{Mosaic attenuation pattern}

This pattern is characterised by a patchwork of intermingled areas of increased and decreased attenuation that

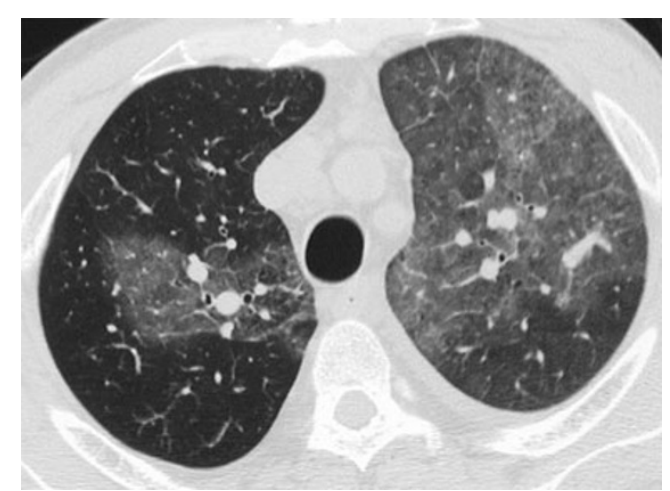

Fig. 45 Chronic thromoboembolic pulmonary arterial hypertension. HRCT at the level of the upper lobes exhibits a "mosaic attenuation pattern" with patchy areas of increased attenuation within which large calibre vessels are seen representing the hyperperfused areas compared with the areas of decreased attenuation with small calibre vessels, which represent the hypoperfused areas (from [45]) 
may represent (1) patchy interstitial disease, (2) obliterative small airways disease and (3) occlusive small vascular disease [4].

Classic entity The classic example of this pattern (as well as of the "decreased attenuation pattern") is obliterative bronchiolitis $(O B)$, as it can also present with patchy areas of decreased attenuation intermingled with areas of increased attenuation. Air-trapping (represented by areas of decreased attenuation) secondary to focal areas of bronchial or bronchiolar obstruction can be accentuated at expiration. Obliterative bronchiolitis can also accompany large airways diseases such as post infection bronchiectasis, cystic fibrosis (Fig. 44) or present as the major pulmonary manifestation of graft-versus-host disease [69].

\section{Mimics Chronic thromboembolic pulmonary hypertension} may present with mosaic attenuation pattern due to patchy vascular hypoxemia. In case of vascular hypoxemia, however, there is significantly reduced calibre of the vessels in areas of decreased attenuation and there is no enhancement of the differing attenuations in expiratory scan compared with "mosaic attenuation" secondary to small airways disease [70] (Fig. 45).

Subacute hypersensitivity pneumonitis may also exhibit a mosaic attenuation pattern, combining both an "interstitial" cause, manifesting as areas of increased attenuation (ground-glass opacity), and a "small airways disease" cause, manifesting as areas of decreased attenuation, which may accentuate on expiratory CT [3]. Respiratory bronchiolitis may also occasionally produce this pattern.

\section{Conclusions}

Evaluation of interstitial lung diseases on HRCT is based on an HRCT-pattern approach to diagnosis, where there should be effort to assign the predominant HRCT pattern to each case. There are various mimics in each HRCT pattern and one entity may have many faces and therefore may mimic many patterns. There may also be possible overlap of HRCT patterns in one case. Moreover, we should keep in mind that no HRCT pattern provides a specific etiological diagnosis. In order to reach a correct and confident diagnosis, one should approach the predominant HRCT pattern by identifying any specific and distinctive imaging clues and try to narrow the differential diagnosis by integrating the HRCT findings with the clinical and laboratory findings.

Open Access This article is distributed under the terms of the Creative Commons Attribution License which permits any use, distribution, and reproduction in any medium, provided the original author(s) and the source are credited.

\section{References}

1. Glaspole IN, du Bois RM, Wells AU (2001) The application of high-resolution CT to diagnosis in diffuse parenchymal lung disease. Monaldi Arch Chest Dis 56:233-239

2. Elicker B, Pereira CA, Webb R, Leslie KO (2008) High-resolution computed tomography patterns of diffuse interstitial lung disease with clinical and pathological correlation. J Bras Pneumol 34:715-744

3. Gotway MB, Reddy GP, Webb WR, Elicker BM, Leung JW (2005) High-resolution CT of the lung: patterns of disease and differential diagnoses. Radiol Clin North Am 43:513-542, viii

4. Hansell DM, Bankier AA, MacMahon H, McLoud TC, Müller NL, Remy J (2008) Fleischner society: glossary of terms for thoracic imaging. Radiology 246:697-722

5. Storto ML, Kee ST, Golden JA, Webb WR (1995) Hydrostatic pulmonary edema: high-resolution CT findings. AJR Am J Roentgenol 165:817-820

6. Johkoh T, Ikezoe J, Tomiyama N, Nagareda T, Kohno N, Takeuchi $\mathrm{N}$, Yamagami H, Kido S, Takashima S, Arisawa J, Kozuka T (1992) CT findings in lymphangitic carcinomatosis of the lung: correlation with histologic findings and pulmonary function tests. AJR Am J Roentgenol 158:1217-1222

7. Criado E, Sánchez M, Ramírez J, Arguis P, de Caralt TM, Perea RJ, Xaubet A (2010) Pulmonary sarcoidosis: typical and atypical manifestations at high-resolution $\mathrm{CT}$ with pathologic correlation. Radiographics 30:1567-1586

8. Hawtin KE, Roddie ME, Mauri FA, Copley SJ (2010) Pulmonary sarcoidosis: the 'great pretender'. Clin Radiol 65:642-650

9. Gawne-Cain ML, Hansell DM (1996) The pattern and distribution of calcified mediastinal lymph nodes in sarcoidosis and tuberculosis: a CT study. Clin Radiol 51:263-267

10. Rodrigues R, Marchiori E, Müller NL (2004) Niemann-pick disease: high-resolution CT findings in two siblings. J Comput Assist Tomogr 28:52-54

11. Arnaud L, Pierre I, Beigelman-Aubry C, Capron F, Brun AL, Rigolet A, Girerd X, Weber N, Piette JC, Grenier PA, Amoura Z, Haroche J (2010) Pulmonary involvement in erdheim-chester disease: a single-center study of thirty-four patients and a review of the literature. Arthritis Rheum 62:3504-3512

12. Nobre LF, Müller NL, de Souza Júnior AS, Marchiori E, Souza IV (2004) Congenital pulmonary lymphangiectasia: CT and pathologic findings. J Thorac Imaging 19:56-59

13. Boland JM, Tazelaar HD, Colby TV, Leslie KO, Hartman TE, Yi ES (2012) Diffuse pulmonary lymphatic disease presenting as interstitial lung disease in adulthood: report of 3 cases. Am J Surg Pathol 36:1548-1554

14. Souza CA, Müller NL, Flint J, Wright JL, Churg A (2005) Idiopathic pulmonary fibrosis: spectrum of high-resolution CT findings. AJR Am J Roentgenol 185:1531-1539

15. MacDonald SL, Rubens MB, Hansell DM, Copley SJ, Desai SR, du Bois RM, Nicholson AG, Colby TV, Wells AU (2001) Nonspecific interstitial pneumonia and usual interstitial pneumonia: comparative appearances at and diagnostic accuracy of thinsection CT. Radiology 221:600-605

16. Elliot TL, Lynch DA, Newell JD Jr, Cool C, Tuder R, Markopoulou K, Veve R, Brown KK (2005) High-resolution computed tomography features of nonspecific interstitial pneumonia and usual interstitial pneumonia. J Comput Assist Tomogr 29:339-345

17. Copley SJ, Wells AU, Sivakumaran P, Rubens MB, Lee YC, Desai SR, MacDonald SL, Thompson RI, Colby TV, Nicholson AG, du Bois RM, Musk AW, Hansell DM (2003) Asbestosis and idiopathic pulmonary fibrosis: comparison of thin-section CT features. Radiology 229:731-736

18. Silva CI, Müller NL, Lynch DA, Curran-Everett D, Brown KK, Lee KS, Chung MP, Churg A (2008) Chronic hypersensitivity 
pneumonitis: differentiation from idiopathic pulmonary fibrosis and nonspecific interstitial pneumonia by using thin-section CT. Radiology 246:288-297

19. Kim EJ, Collard HR, King TE Jr (2009) Rheumatoid arthritisassociated interstitial lung disease: the relevance of histopathologic and radiographic pattern. Chest 136:1397-1405

20. Hartman TE, Primack SL, Swensen SJ, Hansell D, McGuinness G, Müller NL (1993) Desquamative interstitial pneumonia: thinsection CT findings in 22 patients. Radiology 187:787-790

21. Hirschmann JV, Pipavath SN, Godwin JD (2009) Hypersensitivity pneumonitis: a historical, clinical, and radiologic review. Radiographics 29:1921-1938

22. Wells AU, Nicholson AG, Hansell DM, du Bois RM (2003) Respiratory bronchiolitis-associated interstitial lung disease. Semin Respir Crit Care Med 24:585-594

23. Hidalgo A, Franquet T, Giménez A, Bordes R, Pineda R, Madrid M (2006) Smoking-related interstitial lung diseases: radiologicpathologic correlation. Eur Radiol 16:2463-2470

24. Vogel MN, Vatlach M, Weissgerber P, Goeppert B, Claussen CD, Hetzel J, Horger M (2012) HRCT features of pneumocystis jiroveci pneumonia and their evolution before and after treatment in non-HIV immunocompromised patients. Eur J Radiol 81:1315-1320

25. Murch CR, Carr DH (1989) Computed tomography appearances of pulmonary alveolar proteinosis. Clin Radiol 40:240-243

26. McGuinness G, Gruden JF (1999) Viral and pneumocystis carinii infections of the lung in the immunocompromised host. J Thorac Imaging 14:25-36

27. Rossi SE, Erasmus JJ, Volpacchio M, Franquet T, Castiglioni T, McAdams HP (2003) "Crazy-paving" pattern at thin-section CT of the lungs: radiologic-pathologic overview. Radiographics 23: 1509-1519

28. Choi HK, Park CM, Goo JM, Lee HJ (2010) Pulmonary alveolar proteinosis versus exogenous lipoid pneumonia showing crazypaving pattern: comparison of their clinical features and highresolution CT findings. Acta Radiol 51:407-412

29. Collins J, Stern EJ (1997) Ground-glass opacity at CT: the ABCs. AJR Am J Roentgenol 169:355-367

30. Chung MP, Yi CA, Lee HY, Han J, Lee KS (2010) Imaging of pulmonary vasculitis. Radiology 255:322-341

31. Marchiori E, Escuissato DL, Gasparetto TD, Considera DP, Franquet $\mathrm{T}$ (2009) "Crazy-paving" patterns on high-resolution CT scans in patients with pulmonary complications after hematopoietic stem cell transplantation. Korean J Radiol 10:21-24

32. Patsios D, Roberts HC, Paul NS, Chung T, Herman SJ, Pereira A, Weisbrod G (2007) Pictorial review of the many faces of bronchioloalveolar cell carcinoma. Br J Radiol 80:1015-1023

33. Remy-Jardin M, Giraud F, Remy J, Copin MC, Gosselin B, Duhamel A (1993) Importance of ground-glass attenuation in chronic diffuse infiltrative lung disease: pathologic-CT correlation. Radiology 189:693-698

34. Desai SR, Veeraraghavan S, Hansell DM, Nikolakopolou A, Goh NS, Nicholson AG, Colby TV, Denton CP, Black CM, du Bois RM, Wells AU (2004) CT features of lung disease in patients with systemic sclerosis: comparison with idiopathic pulmonary fibrosis and nonspecific interstitial pneumonia. Radiology 232:560-567

35. Devaraj A, Wells AU, Meister MG, Corte TJ, Wort SJ, Hansell DM (2010) Detection of pulmonary hypertension with multidetector CT and echocardiography alone and in combination. Radiology 254:609-616

36. Heyneman LE, Ward S, Lynch DA, Remy-Jardin M, Johkoh T, Müller NL (1999) Respiratory bronchiolitis, respiratory bronchiolitisassociated interstitial lung disease, and desquamative interstitial pneumonia: different entities or part of the spectrum of the same disease process? AJR Am J Roentgenol 173:1617-1622

37. Hartman TE, Primack SL, Kang EY, Swensen SJ, Hansell DM, McGuinness G, Müller NL (1996) Disease progression in usual interstitial pneumonia compared with desquamative interstitial pneumonia. Assessment with serial CT. Chest 110:378-382

38. Brauner MW, Lenoir S, Grenier P, Cluzel P, Battesti JP, Valeyre D (1992) Pulmonary sarcoidosis: CT assessment of lesion reversibility. Radiology 182:349-354

39. Akira M (2002) High-resolution CT in the evaluation of occupational and environmental disease. Radiol Clin North Am 40:43-59

40. Remy-Jardin M, Remy J, Farre I, Marquette CH (1992) Computed tomographic evaluation of silicosis and coal workers' pneumoconiosis. Radiol Clin North Am 30:1155-1176

41. Raoof S, Amchentsev A, Vlahos I, Goud A, Naidich DP (2006) Pictorial essay: multinodular disease: a high-resolution CT scan diagnostic algorithm. Chest 129:805-815

42. Hong SH, Im JG, Lee JS, Song JW, Lee HJ, Yeon KM (1998) High resolution CT findings of miliary tuberculosis. J Comput Assist Tomogr 22:220-224

43. Voloudaki AE, Tritou IN, Magkanas EG, Chalkiadakis GE, Siafakas NM, Gourtsoyiannis NC (1999) HRCT in miliary lung disease. Acta Radiol 40:451-456

44. Akira M, Kitatani F, Lee YS, Kita N, Yamamoto S, Higashihara T, Morimoto S, Ikezoe J, Kozuka T (1988) Diffuse panbronchiolitis: evaluation with high-resolution CT. Radiology 168:433-438

45. Aquino SL, Gamsu G, Webb WR, Kee ST (1996) Tree-in-Bud pattern: frequency and significance on thin section CT. J Comput Assist Tomogr 20:594-599

46. Im JG, Itoh H, Shim YS, Lee JH, Ahn J, Han MC, Noma S (1993) Pulmonary tuberculosis: CT findings - early active disease and sequential change with antituberculous therapy. Radiology 186: 653-660

47. Koh WJ, Lee KS, Kwon OJ, Jeong YJ, Kwak SH, Kim TS (2005) Bilateral bronchiectasis and bronchiolitis at thin-section CT: diagnostic implications in nontuberculous mycobacterial pulmonary infection. Radiology 235:282-288

48. Rossi SE, Franquet T, Volpacchio M, Giménez A, Aguilar G (2005) Tree-in-bud pattern at thin-section CT of the lungs: radiologic-pathologic overview. Radiographics 25:789-801

49. Franquet T, Müller NL, Lee KS, Oikonomou A, Flint JD (2005) Pulmonary candidiasis after hematopoietic stem cell transplantation: thin-section CT findings. Radiology 236:332-337

50. Franquet T, Müller NL, Oikonomou A, Flint JD (2004) Aspergillus infection of the airways: computed tomography and pathologic findings. J Comput Assist Tomogr 28:10-16

51. Franquet T (2011) Imaging of pulmonary viral pneumonia. Radiology 260:18-39

52. Franquet T, Gimenez A, Prats R, Rodriguez-Arias JM, Rodriguez C (2002) Thrombotic microangiopathy of pulmonary tumors: a vascular cause of tree-in-bud pattern on CT. AJR Am J Roentgenol 179:897-899

53. Baldwin CI, Todd A, Bourke S, Allen A, Calvert JE (1998) Pigeon fanciers' lung: effects of smoking on serum and salivary antibody responses to pigeon antigens. Clin Exp Immunol 113:166-172

54. Furuiye M, Miyake S, Miyazaki Y, Ohtani Y, Inase N, Umino T, Yoshizawa Y (2007) Effect of cigarette smoking on the development of murine chronic pigeon breeder's lung. The difference between a short-term and a long-term exposure. J Med Dent Sci 54:87-95

55. Desai SR, Ryan SM, Colby TV (2003) Smoking-related interstitial lung diseases: histopathological and imaging perspectives. Clin Radiol 58:259-268

56. Oikonomou A, Hansell DM (2002) Organizing pneumonia: the many morphological faces. Eur Radiol 12:1486-1496

57. Cordier JF (2000) Organizing pneumonia. Thorax 55:318-328

58. Johkoh T, Müller NL, Pickford HA, Hartman TE, Ichikado K, Akira M, Honda O, Nakamura H (1999) Lymphocytic interstitial pneumonia: thin-section CT findings in 22 patients. Radiology 212:567-572 
59. Howling SJ, Hansell DM, Wells AU, Nicholson AG, Flint JD, Müller NL (1999) Follicular bronchiolitis: thin-section CT and histologic findings. Radiology 212:637-642

60. Hansell DM, Milne DG, Wilsher ML, Wells AU (1998) Pulmonary sarcoidosis: morphologic associations of airflow obstruction at thin-section CT. Radiology 209:697-704

61. Newman LS, Buschman DL, Newell JD Jr, Lynch DA (1994) Beryllium disease: assessment with CT. Radiology 190:835-840

62. Johnson SR, Cordier JF, Lazor R, Cottin V, Costabel U, Harari S, Reynaud-Gaubert M, Boehler A, Brauner M, Popper H, Bonetti F, Kingswood C, Review Panel of the ERS LAM Task Force (2010) European respiratory society guidelines for the diagnosis and management of lymphangioleiomyomatosis. Eur Respir J 35:14-26

63. Silva CI, Flint JD, Levy RD, Müller NL (2006) Diffuse lung cysts in lymphoid interstitial pneumonia: high-resolution CT and pathologic findings. J Thorac Imaging 21:241-244

64. Murata K, Itoh H, Todo G, Kanaoka M, Noma S, Itoh T, Furuta M, Asamoto H, Torizuka K (1986) Centrilobular lesions of the lung: demonstration by high-resolution CT and pathologic correlation. Radiology 161:641-645
65. Marchiori E, Müller NL, Soares Souza A Jr, Escuissato DL, Gasparetto EL, Franquet T (2005) Pulmonary disease in patients with AIDS: high-resolution CT and pathologic findings. AJR Am J Roentgenol 184:757-764

66. Franquet T, Hansell DM, Senbanjo T, Remy-Jardin M, Müller NL (2003) Lung cysts in subacute hypersensitivity pneumonitis. J Comput Assist Tomogr 27:475-478

67. Hansell DM (2001) HRCT of obliterative bronchiolitis and other small airways diseases. Semin Roentgenol 36:51-65

68. Copley SJ, Wells AU, Müller NL, Rubens MB, Hollings NP, Cleverley JR, Milne DG, Hansell DM (2002) Thin-section CT in obstructive pulmonary disease: discriminatory value. Radiology 223:812-819

69. Worthy SA, Flint JD, Müller NL (1997) Pulmonary complications after bone marrow transplantation: high-resolution CT and pathologic findings. Radiographics 17:1359-1371

70. Oikonomou A, Dennie CJ, Müller NL, Seely JM, Matzinger FR, Rubens FD (2004) Chronic thromboembolic pulmonary arterial hypertension: correlation of postoperative results of thromboendarterectomy with preoperative helical contrast-enhanced computed tomography. J Thorac Imaging 19:67-73 